\title{
Thermocapillary Convection in Floating Zones
}

This paper provides an overview of ongoing studies in the area of thermocapillary convection driven by a surface tension gradient parallel to the free surface in a floating zone. Here, research interests are focused around the onset of oscillatory thermocapillary convection, also known as the transition from quasisteady convection to oscillatory convection. The onset of oscillation depends on a set of critical parameters, and the margin relationship can be represented by a complex function of the critical parameters. The experimental results indicate that the velocity deviation of an oscillatory flow has the same order of magnitude as that of an average flow, and the deviations of other quantities, such as temperature and free surface radii fluctuations, are much smaller when compared with their normal counterparts. Therefore, the onset of oscillation should be a result of the dynamic process in a fluid, and the problem is a strongly nonlinear one. In the past few decades, several theoretical models have been introduced to tackle the problem using analytical methods, linear instability analysis methods, energy instability methods, and unsteady 3D numerical methods. The last of the above mentioned methods is known to be the most suitable for a thorough analysis of strong nonlinear processes, which generally leads to a better comparison with the experimental results. The transition from oscillatory thermocapillary convection to turbulence falls under the studies of chaotic behavior in a new system, which opens a fascinating new frontier in nonlinear science, a hot research area drawing many recent works. This paper reviews theoretical models and analysis, and also experimental research, on thermocapillary connection in floating zones. It cites 93 references. [DOI: 10.1115/1.2820798]

\section{Introduction}

Space environment utilizations, especially in manned space flights, are operated under the microgravity condition, where the capillary effects plays a dominant role, and the gravitational effects-such as buoyancy convection, sedimentation, and pressure gradient due to the earth gravity - are greatly reduced. Living and working in a manned space structure, such as a space shuttle or a space station require a good understanding of fluid behavior under microgravity conditions, for a system can be quite different from that on the ground. Some background discussion on this can be found in a review paper [1]. Various branches in microgravity engineering science, such as fluid management, heat management, fire safety, and multiphase flow related to life support systems in a microgravity environment, are expanding rapidly as more and more requirements are flooding in from many manned space missions.

The microgravity environment offers an excellent platform for scientific researches and applications, as those described, for example, in the books of collected papers [2-4]. Natural convection induced by the nonuniformity of surface tension on a gas/liquid free surface or liquid/liquid interface is important in mass and heat transfer in many processes in the microgravity environment. The surface tension depends on such factors as temperature, solutal concentration, electric potential, etc., and the surface tension gradient is the result of the gradient of the physical quantities. On the ground, other than small-scale systems such as the flow in a film or the migration of a small fluid drop, where the process is dictated by the surface tension gradient, the gravitational effects such as buoyancy are dominating factors in most of the processes. Convection induced by the temperature gradient and driven by the surface tension gradient on the free surface is called a thermocapillary convection, which is the centerpiece of the discussion in the present paper.

Another type of convection under microgravity conditions is

Published online March 5, 2008. Transmitted by Assoc. Editor T. Y. Fan. the Marangoni convection, where the temperature gradient is perpendicular to the free surface. Pearson [5] was the first to study the instability in a liquid layer bounded by a free surface and a heated solid plane boundary in the microgravity environment. $\mathrm{He}$ demonstrated that there was an onset of convection if the applied temperature difference between the solid boundary and the free surface boundary was above a critical value. The analysis of Pearson flow under microgravity conditions is similar to that of Benard-Rayleigh convection on the ground if the differences in their mechanisms are disregarded. Both the thermocapillary convection and the Marangoni convection are fundamental types of convection in microgravity conditions, and the thermocapillary convection is discussed in the present paper.

The floating zone technique is a zone refining method for the purification of crystals, primarily used for high-quality semiconductor crystal growth since the early 1950s. The main advantage of the floating zone technique is not needing a container during the crystal growth, while the main limitation on the ground is the level of difficulty of manufacturing single, larger scale crystals due to easy breakage of the liquid bridge by gravity. This limitation disappears under microgravity conditions, making the floating zone technique one of the most promising methods in space materials processing. Thermocapillary convection in floating zones has become one of the mainstream subjects in microgravity fluid physics and space materials science.

A floating zone model is introduced to explain the behavior of a liquid bridge confined by a free surface and two coaxial boundaries related to the solidification and melting of liquid/solid interfaces, as shown in Fig. 1. Two interfaces separating the liquid region and the solid region are curved during the materials processing, and the complete theoretical problem can be solved by matching solutions in the liquid bridge and the two solid regions [6]. Affected by gravity at the ground level, the free surface configuration of a floating zone is in the shape of calabash, as shown in Figs. $1(a)-1(c)$, which is different from that under microgravity conditions, where it is symmetric to the middle plane of the liquid bridge in the longitudinal direction, as shown in Figs. $1(d)$ and 


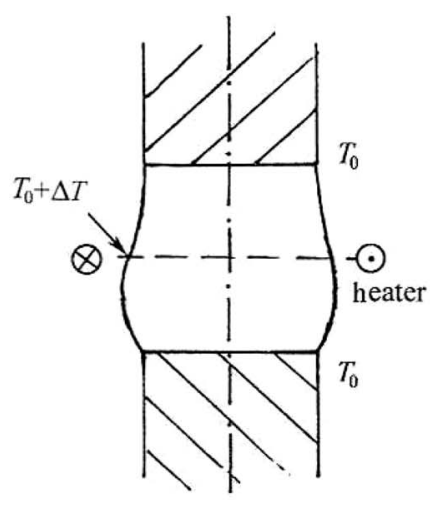

(a)

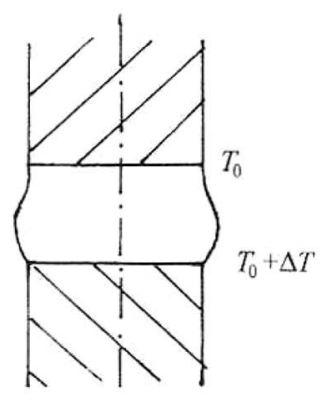

(b)

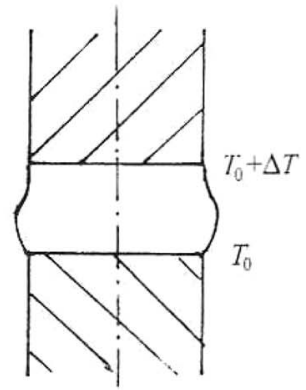

(c)
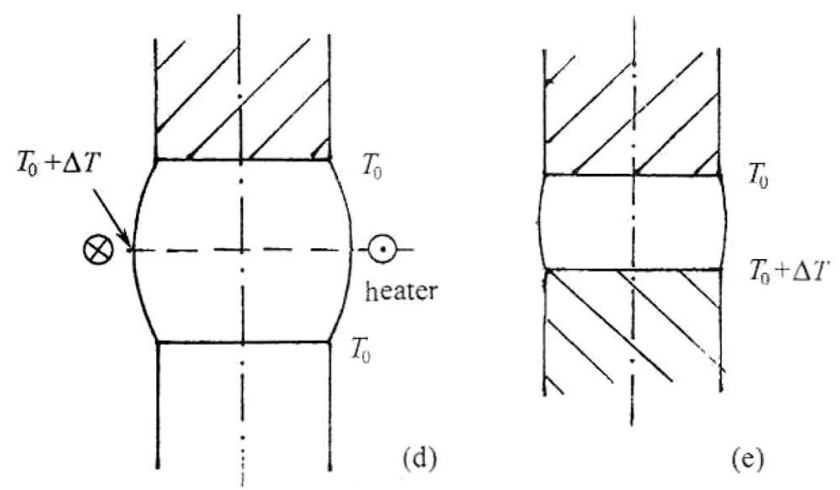

(e)

Fig. 1 Typical floating zone models. (a) Floating full zone on the ground. (b) Floating half zone heated from bottom on the ground. (c) Floating half zone heated from upper on the ground. (d) Floating full zone in the microgravity environment. (e) Floating half zone in the microgravity environment.

1(e). Many studies have simplified the problem by assuming a cylindrical liquid bridge. The convection in a full floating zone was first suggested by Chang and Wilcox [7]. A floating half zone is composed of one-half of a full floating zone dissected from the heater plane and assumes a higher constant temperature at the cutting plane, as shown in Figs. 1(b), 1(c), and 1(e). Here, an applied temperature difference between two rods can result in a temperature gradient, which subsequently generates a surface tension gradient on the free surface. The nonuniformity of surface tension will then drive the thermocapillary convection in the liquid bridge. The floating half zone model was originated by Chun and Wuest [8,9] and Schwabe et al. [10,11], and has been adopted broadly since its introduction. There are several examples documented in books $[12,13]$. The thermal stable model of an upper rod heated as shown in Fig. 1(c) is usually analyzed on the ground. A typical hydrodynamic model of the floating half zone, with both interfaces assumed to be planar, is displayed in Fig. 2. A better understanding of thermocapillary convection in the floating zone is crucial for further advancement in both applications of space materials processing and the microgravity fluid physics theories dealing with thermocapillary convection mechanism.

It is worth noting that, with regard to the dominant surface tension effect, the similarity between the microflow on the ground and the large-scale flow in microgravity conditions has inspired some researchers to compare these two processes. The expectation is that some understanding of microflow phenomena can be obtained by simulating a large-scale flow in the microgravity condition, which is a relatively easier experiment to observe.

In the present paper, we concentrate mostly on the model of floating half zone, namely, the model shown in Fig. 1(c) on the ground and that in Fig. 1(e) in the microgravity condition. The next section discusses the critical parameters for the onset of os- cillatory thermocapillary convection, with the margin relationship presented as a complex function depending on the critical parameters. The experimental results for both small Prandtl number fluids $(\operatorname{Pr} \ll 1)$ and larger Prandtl number fluids $(\operatorname{Pr}>1)$ are put together in Sec. 3. We have limited experimental results in space, especially for the case of small Prandtl number fluids. The theoretical models are summarized in Sec. 4, and the results of different numerical codes are comparable for the same Prandtl number, aspect ratio, and ratio of liquid bridge volume in the case of small Prandtl numbers. The changes from oscillatory thermocapillary convection to turbulence, which have caught research attention recently, are briefly discussed in Sec. 5 . We summarize the study and present our conclusions in the last section.

\section{Simulation Parameters}

The conservations of mass, momentum, and energy in a liquid bridge in addition to suitable boundary conditions at the free surface and the solid rods are satisfied for a typical hydrodynamic model of the floating zone convection. The concentration conservation is involved sometimes in the process of crystal growth in a floating zone, and the complete problem could be solved by coupling the conservation equations in the liquid bridge and the energy equation in the solid regions [6]. From the macroscopic viewpoint of hydrodynamics, the fluid of the liquid bridge is considered as a continuous medium, and the microscopic phenomena, such as solidification and melting processes at the liquid/solid interface, are macroscopically described by the concentration and heat boundary conditions.

The physical model of a floating half zone consists usually of a liquid bridge floating between two coaxial rods of the same radius $r_{0}$ and height $l$. A cylindrical coordinate system $(r, \theta, z)$ is used, as 


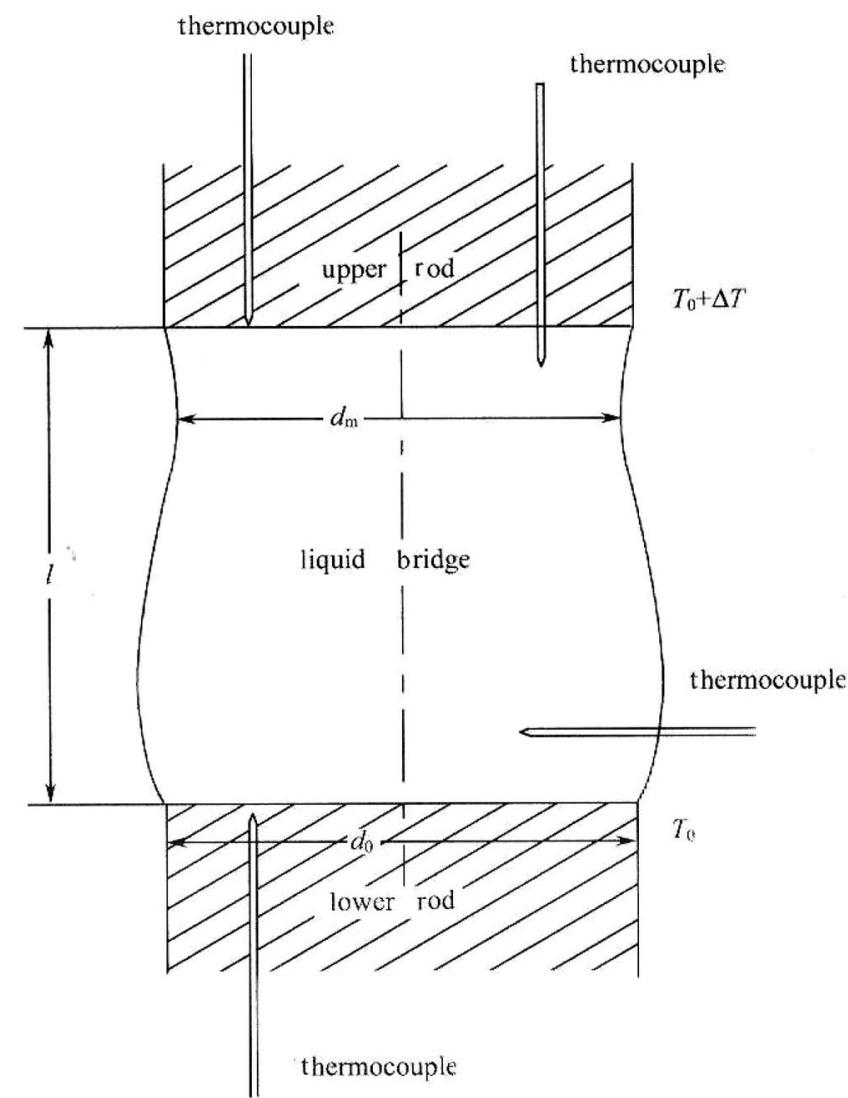

Fig. 2 The schematic diagram of the floating half zone convection

shown in Fig. 2. A temperature difference $\Delta T$ is applied between the top rod and the bottom rod. The fluid of the liquid bridge is treated as an incompressible Newtonian liquid, and the Boussinesq approximation is often used.

The nondimensional quantities are defined as follows: the time $t$, coordinates $(r, z)$, and velocity $\mathbf{v}$ are respectively divided by $l / \mathbf{v}^{*}, l$, and $\mathbf{v}^{*}$; the temperature and pressure are divided respectively by $\Delta T$ and $\rho \mathbf{v}^{* 2}$; and the body force is divided by $\rho \mathbf{v}^{* 2} / l$, where the typical velocity $\mathbf{v}^{*}=\left|\sigma_{T}^{\prime}\right| \Delta T /(\rho \nu)$ comes from the equilibrium of the surface tension gradient $\sigma_{T}^{\prime}$ and the viscous stress. Then, the nondimensional equations are

$$
\begin{gathered}
\nabla \cdot \bar{V}=0 \\
\frac{\partial \bar{V}}{\partial \tau}+\bar{V} \cdot \nabla \bar{V}=-\nabla P+\frac{1}{\operatorname{Re}} \nabla^{2} \bar{V}+\bar{F} \\
\frac{\partial \Theta}{\partial \tau}+\bar{V} \cdot \nabla \Theta=\frac{1}{\mathrm{Ma}} \nabla^{2} \Theta
\end{gathered}
$$

where $\operatorname{Re}=\mathbf{v}^{*} l / \nu$ is the Reynolds number, and a new nondimensional parameter, the Marangoni number, is given as

$$
\mathrm{Ma}=\frac{\nu^{*} \ell}{\kappa}=\frac{\left|\sigma_{T}^{\prime}\right| \Delta T \ell}{\rho \kappa \nu}
$$

The coefficients $\kappa$ and $\nu$ are, respectively, the thermal diffusivity and the kinematic viscosity, and $\rho$ is the liquid density. The concentration equation can be given in a form similar to Eq. (3). The gravitational force $\rho \mathbf{g}$ or the buoyant force $\rho \beta_{T}\left(T-T_{0}\right) \mathbf{g}$ involves the term of the body force $\mathbf{F}$ of Eq. (2) and is omitted in the microgravity environment. Here, $\beta_{T}$ is the thermal expansion coefficient.

The natural convection is driven mainly by the buoyancy on the ground and by the capillary force on the free surface or interface in the microgravity environment. To compare the features of typical flows, Table 1 lists the nondimensional parameters for the viscous flow, buoyancy convection, and thermocapillary convection, and Re, Pe, Pr, Gr, Ra, and Ma are, respectively, the Reynolds number, Peclet number, Prandtl number, Grashof number, Rayleigh number, and Marangoni number. The nondimensional parameters describe the relative importance of the effects in the hydrodynamic processes. The Prandtl number and Marangoni number, or related Reynolds number or Peclet number, are critical parameters for many dynamic processes in the microgravity environment.

The momentum boundary conditions require the stress component to be balanced by the capillary force related to the surface tension in the normal direction (Laplace relationship) and related to the surface tension gradient in the tangential direction. Therefore, the crispation number $\mathrm{Cr}$ is defined as

$$
C r_{t}=\frac{\left|\sigma_{T}^{\prime}\right| \Delta T}{\sigma} \quad \text { or } \quad C r_{s}=\frac{\left|\sigma_{c}^{\prime}\right| \Delta C}{\sigma}
$$

There is the condition $C r \ll 1$ in most cases. It means that the free surface has a relative "solidity" in some sense, and the displacement deviation according to the capillary effect on the free surface is smaller in comparison with the typical scale in the dynamic process.

Another nondimensional parameter in the microgravity fluid physics is the Bond number, which is the ratio of the gravity effect and the surface tension effect and is defined as

$$
B_{0}=\frac{\rho g \ell^{2}}{\sigma} \text { or } B_{d}=\frac{B_{0}}{C r}
$$

Parameters $B_{0}$ and $B_{d}$ are the static Bond number and the dynamic Bond number, respectively. The density $\rho$ may be displayed by the density difference $\Delta \rho$ if the buoyancy effect is emphasized. The Bond number is small in the microgravity environment of low $g$, small scale $l$, and/or small density difference $\Delta \rho$. Therefore, the effects related to low gravity can be simulated by reducing the typical scale $l$ or the density difference $\Delta \rho$ on the ground. This is the basis of small-scale experiments and neutral floating experiments on the ground to simulate some processes in the microgravity phenomena.

Thermocapillary convection occurs if a temperature difference is applied on the free surface. Until now, the most emphasized problems on thermocapillary convection are related to the onset of oscillation, that is, the transition from the laminar convection to the oscillatory convection, and it is an important subject in the fluid mechanics. From the viewpoint of materials science, the on-

Table 1 Typical nondimensional parameters

\begin{tabular}{lccc}
\hline \hline & Viscous flow & Buoyancy convection & Thermocapillary convection \\
\hline Typical velocity & $\mathbf{v}_{0}$ & $\mathbf{v}^{*}=g \beta_{T} \Delta T l^{2} / v$ & $\mathbf{v}^{*}=-\sigma_{T}^{\prime} \Delta T / \rho \nu$ \\
Reynolds number & $\operatorname{Re}=\mathbf{v}_{0} l / \nu$ & $\mathrm{Gr}=\mathbf{v}^{*} l / \nu$ & $\operatorname{Re}=\mathbf{v}^{*} / \nu=\mathrm{Ma} / \operatorname{Pr}$ \\
Peclet number & $\operatorname{Pe}=\mathbf{v}_{0} l / \kappa=\operatorname{RePr}$ & $\operatorname{Ra}=\mathbf{v}^{*} l / \kappa=\mathrm{GrPr}$ & $\mathrm{Ma}=\mathbf{v}^{*} l / \kappa=\operatorname{RePr}$ \\
Prandtl number & $\operatorname{Pr}=\nu / \kappa=\operatorname{Pe} / \operatorname{Re}$ & $\operatorname{Pr}=\nu / \kappa=\operatorname{Ra} / \mathrm{Gr}$ & $\operatorname{Pr}=\nu / \kappa=\mathrm{Ma} / \operatorname{Re}$ \\
\hline \hline
\end{tabular}


set of oscillatory convection relates to the appearance of striation in a crystal during solidification in the floating zone processing, and avoiding striation is important in controlling the crystal quality. During the increase of the applied temperature difference $\Delta T$, the oscillatory thermocapillary convection occurs if the temperature difference is larger than a critical temperature difference $(\Delta T)_{c}$, which is related to a critical Marangoni number $(\mathrm{Ma})_{c}$. Therefore, the Marangoni number is a sensitive critical parameter for the transition process [8-11].

Most experiments on floating zones on the ground are based on the small Bond number simulation by using a small scale, and a transparent liquid, which Prandtl number is large, will be helpful for the optical diagnostics. However, the behavior of heat transfer on a liquid bridge with large Prandtl numbers is different from that of the nontransparent medium, such as the metals and alloys of small Prandtl numbers. The Prandtl number is another critical parameter for the onset of oscillatory convection, and has been studied extensively (see, for example, Ref. [14]).

It is noticed that the onset of oscillatory convection depends on the geometric features of the liquid bridge, and the aspect ratio $A=l / r_{0}$ is introduced as a geometrical critical parameter. The critical Marangoni number in space experiments of larger scale in the microgravity environment gave a much larger value than the one in the experiments of small scale on the ground for the same geometrical aspect ratio. The reason is probably due to the more important effect of the boundary layer in a liquid bridge of smaller scale of a few millimeters in diameters; the diameters of liquid bridges in the space experiments are several centimeters [15].

The liquid bridges in many models are considered as cylindrical configurations; however, a cylindrical configuration cannot be kept on the one- $g$ condition and is only a special case in the microgravity condition. The results show that the margin curves separate onto two branches, and the critical Marangoni number increases or decreases with the increasing liquid bridge volume for a slender or a fat liquid bridge, respectively, for a fixed geometrical configuration of the liquid bridge. The critical Marangoni numbers may have difference of $100 \%$ or $200 \%$ for cases of the same aspect ratio but different liquid bridge volumes. The results show that the liquid bridge volume $V$ is a sensitive critical parameter for the onset of oscillatory thermocapillary convection. Therefore, the experimental results should deal with a given liquid bridge volume; otherwise, the results may have very large dispersion and may be difficult to compare due to different liquid bridge volumes. The nondimensional liquid bridge volume ratio $V / V_{0}$ may be introduced, where $V_{0}=\pi d_{0}^{2} l / 4$ is a cylindrical liquid bridge with the same aspect ratio, and the discussions will be given in the next section in detail $[16,17]$.

Furthermore, the heat transfer process described by the Biot number $\mathrm{Bi}=q d / k$ and Nusselt number are also the critical parameters for the onset of oscillation, where $q$ and $k$ are the heat exchange and thermal conductivity at the free surface, respectively. In summary, the critical parameters for the onset of oscillatory thermocapillary convection may be classified as follows.

(1) geometrical features: aspect ratio $A$, height of liquid bridge $l$, and ratio of liquid bridge volume $V / V_{0}$

(2) property of liquid medium: Prandtl number Pr and Peclet number Pe

(3) dynamic parameters: Marangoni number Ma or Reynolds number Re and Grashof number Gr in gravitational conditions

(4) heat transfer from the liquid bridge region: Biot number Bi and Nusselt number $\mathrm{Nu}$

The research purpose on the onset of oscillatory thermocapillary convection is to find the critical condition,

$$
F\left(A, V / V_{0}, l ; \mathrm{Pr}, \mathrm{Pe} ; \mathrm{Ma}, \mathrm{Gr} ; \mathrm{Bi}, \mathrm{Nu} ; \ldots\right)=0
$$

The experiments have been performed mostly in cases of small liquid bridge of large Prandtl number, and are limited in cases of small Prandtl number fluids and in the microgravity environment. The theoretical studies are given by the methods of instability analyses, energy method, and unsteady and 3D numerical calculations for a cylindrical liquid bridge in most cases and for the curved free surface of liquid bridge recently.

\section{Experimental Results}

A great number of experimental studies have been devoted to thermocapillary convection in the floating zone; however, most of them are performed for the transparent liquids, which is easy for optical diagnostics. The liquid bridges of the space materials processing, such as semiconductors, metals, and alloys, are related to the behavior of liquids with small Prandtl numbers $\operatorname{Pr} \ll 1$. The liquid bridge of the transparent liquid with usually a large Prandtl number $\operatorname{Pr}>1$ is convenient for experimental arrangement, but the behavior of heat transfer in cases of $\operatorname{Pr} \ll 1$ is difficult to be simulated quite well by that in cases of $\operatorname{Pr}>1$. It is noted that the mechanism on the onset of oscillatory thermocapillary convection in the liquid bridge of small Prandtl numbers $\operatorname{Pr} \ll 1$ is different from that of large Prandtl numbers $\operatorname{Pr}>1$ [18].

3.1 Experiments for Small Prandtl Number Fluids $\operatorname{Pr} \ll 1$. Difficulties in experiments on a liquid bridge with $\operatorname{Pr} \ll 1$ include the flow visualization due to nontransparency and the surface tension change due to the free surface pollution. An experiment for small Prandtl numbers in the microgravity environment is much difficult than the one on the ground, and the space experiment on the thermocapillary convection in case of small Prandtl numbers is extremely limited until now [25]. Nakamura et al. [19] studied the oscillatory features of thermocapillary convection in the melting silicon liquid bridge on the ground and on board the sounding rocket. The Marangoni numbers in their experiments were much larger than the critical Marangoni number, and the process on the onset of oscillation was not a concern.

Levenstam and Amberg [18] found theoretically that there are two bifurcation transitions in the liquid bridge, that is, the transition of the steady and axisymmetric flow to the steady and nonaxisymmetric convection at first, and that to the onset of oscillatory thermocapillary convection in the cases of small Prandtl number fluids. Therefore, there are two critical Marangoni numbers $(\mathrm{Ma})_{c 1}$ and $(\mathrm{Ma})_{c 2}$ related respectively to the two transitions. The first transition feature has not been proven yet by experiments. The experiments on crystal growth in a floating zone discover that the transition from striation free to striation in the crystal growth during the increase of the applied temperature difference is associated with the onset of oscillatory flow in the melt. This feature can determine experimentally the second transition condition and the critical Marangoni number $(\mathrm{Ma})_{c 2}$. Table 2 lists the experimental results of critical Marangoni numbers $(\mathrm{Ma})_{c 2}$ in the floating full zone for the onset of oscillation in the case of small Prandtl number fluids. It seems that the buoyancy effect is important in cases of experiments on the ground because of the relatively larger scales and then the larger Bond numbers. The results of critical Marangoni numbers $(\mathrm{Ma})_{c 2}$ disperse from 100-200 to more than 1000, which are much larger than the one given by the theoretical expectation, and summarized experimental results are given as [25]

$$
(\mathrm{Ma})_{c 2}=2.2 \times 10^{4} \operatorname{Pr}^{1.32}
$$

Some conditions of these experiments are not described clearly, for example, the pollution on the free surface and the influence of the liquid bridge volume.

The experimental models of the floating half zone in cases of small Prandtl number liquids avoid the solidification process, and these simplified models can satisfy more controllable experimen- 
Table 2 Experimental results of the floating full zone in cases of $\operatorname{Pr} \ll 1$

\begin{tabular}{lcccccr}
\hline \hline Materials & Pr & Height $(\mathrm{mm})$ & Diameter $(\mathrm{mm})$ & $(\mathrm{Ma})_{c}$ & $f_{c}(\mathrm{~Hz})$ & Ref. \\
\hline $\mathrm{Ti}_{3} \mathrm{Cu}$ & - & 12.0 & 6.0 & - & 0.03 & 20 \\
$\mathrm{Si}$ & 0.02 & 14.0 & 10.0 & $100-200$ & $\sim 1$ & 21 \\
$\mathrm{Mo}$ & 0.025 & 3.4 & 2.6 & 638 & $\sim 1$ & 22 \\
& & 4.1 & 4.0 & 925 & $\sim 1$ & 22 \\
& & 5.1 & 6.0 & 1625 & $\sim 1$ & 22 \\
$\mathrm{Nb}$ & 0.025 & 4.0 & 6.0 & 1025 & $\sim 1$ & 23 \\
$\mathrm{GaAs}$ & 0.068 & 7.6 & 6.0 & $400-500$ & $\sim 1$ & 24 \\
$\mathrm{GaSb}$ & 0.0166 & 31.5 & 18.0 & $375 \pm 125$ & $0.034-0.055$ & $25^{\mathrm{a}}$ \\
\hline \hline
\end{tabular}

${ }^{\mathrm{a}}$ Space experiments in STS-77.

tal conditions. Han and co-workers [26,27] studied experimentally the thermocapillary convection in a mercury liquid bridge and discussed the onset of oscillatory thermocapillary convection in a floating half zone formed by a small Prandtl number liquid for the first time. An optical diagnostic of a noncontacted method based on the free surface oscillation related to the temperature oscillation in the liquid bridge, which is used to measure the transition process for a transparent liquid [28,29], was applied to a system of nontransparent mercury liquid bridge with $3 \mathrm{~mm}$ in diameter and an adjusted height. The critical Marangoni number and the critical frequency for a liquid bridge of aspect ratio $A=0.6$ and ratio of the liquid bridge volume $V / V_{0}=0.6$ are measured as follows:

$$
(\mathrm{Ma})_{c 2}=900 f_{c}=5 \mathrm{~Hz}
$$

The pollution films easily appear on the free surface of a liquid bridge in the case of the small Prandtl number fluids, and may change the surface tension and then the critical Marangoni number. The critical Marangoni number given above is probably larger than that for a liquid bridge without films. Yang and Kou [30] completed a similar floating half zone experiment for a tin liquid bridge of $4 \mathrm{~mm}$ in height and $4 \mathrm{~mm}$ in diameter, and they obtained the following critical parameters:

$$
(\mathrm{Ma})_{c 2}=194 \pm 14 f_{c}=1.1 \pm 0.1 \mathrm{~Hz}
$$

The results were determined experimentally by the onset of temperature oscillation measured by a $J$-type thermocouple contacted with the free surface [30]. This result and other experimental results were summarized by an empirical formula,

$$
(\mathrm{Ma})_{c 2}=2000 \operatorname{Pr}^{0.6} 10^{-2}<\operatorname{Pr}<10^{2}
$$

Takagi et al. [31] performed the same experiment on a floating half zone consisting of molten tin, and the onset of temperature oscillation at the free surface was measured by a noncontacted system of radiation thermometer. The critical parameters are given as follows:

$$
(\mathrm{Ma})_{c 2}=43.3 f_{c}=0.08 \mathrm{~Hz}
$$

for a liquid bridge of $2.97 \mathrm{~mm}$ in height and $3 \mathrm{~mm}$ in diameter. In comparison with both experiments, it shows that the contacted measurement may greatly change the oscillatory feature especially in a liquid bridge of small Prandtl number.

Limited experiments on the small Prandtl number liquid bridge of the floating half zone have been performed on the ground due to the difficulties, and there is no schedule of space experiment to be arranged at the present time and in the near future. More studies need to be devoted to learning the thermocapillary behavior of small Prandtl number liquids.

3.2 Experiments for Medium and Large Prandtl Number Fluids $\operatorname{Pr}>1$. A lot of experiments have been devoted to thermocapillary convection in a liquid bridge in the condition $\operatorname{Pr}>1$ since the beginning of the research [8-11], and the recent developments were reviewed in Refs. $[13,14,32]$. Most experimental studies have been completed on the ground in the small liquid bridges of a few millimeters in diameter based on the simulation of small Bond numbers, Bo $<1$.

The preliminary experimental researches on thermocapillary convection in the floating half zone deal with the critical conditions for the onset of oscillation: For example, the critical Marangoni number depends on the Prandtl number, aspect ratio, velocity and temperature distributions on the free surface, etc. Typical facilities of these experiments consist of a particle image velocimetry (PIV) system to observe the flow patterns in a vertical cross section and a thermocouple system to record the temperature in the liquid bridge or near the free surface. The onset of oscillation is shown by the transition of flow patterns from axisymmetric to nonsymmetric and the transition of temperature at a fixed point of the liquid bridge from smooth variation to the oscillation. A summary of these experiments may be seen, for example, in Ref. [15]. The transparency of the liquid bridge is a benefit to the optical measurements, and the fluids of large Prandtl numbers reduce the disturbed influence due to the inserted thermocouple of temperature measurement $[28,29]$. The influence is relatively quite larger in cases of small Prandtl number fluids, as discussed in Sec. 3.1 .

Experimental studies have been performed for fluids of different Prandtl numbers, for example, the $\mathrm{KCl}$ of $\mathrm{Pr}=1.025$ [33], the methyl alcohol of $\mathrm{Pr}=6.8$ [9], the $\mathrm{NaNO}_{3}$ of $\mathrm{Pr}=7.05$ [33], the octadecane of $\operatorname{Pr}=18.7$ [34], the hexadecane $\mathrm{C}_{16} \mathrm{H}_{34}$ of $\mathrm{Pr}=42$ [35], the tetracosane $\mathrm{C}_{24} \mathrm{H}_{50}$ of $\mathrm{Pr}=49$ [33], and various silicon oils covering a range of $\operatorname{Pr}=O\left(10^{1}\right)-O\left(10^{2}\right)$, such as the $10 \mathrm{cS}$ silicon oil of $\operatorname{Pr}=108$, which is often used in thermocapillary experiments due to its chemical stable [28,29]. The experimental results show that the critical Marangoni number increases nearly linearly from $10^{3}$ to $10^{4}$ when the Prandtl number increases from $10^{0}$ to $10^{2}$, as described in relationships (8) and (9).

The Bond number is usually $B_{0}=O\left(10^{\circ}\right)$ in order of magnitude in the experiments of small scale on the ground, and the effect of buoyancy cannot be completely omitted. The Bond number can be much smaller than 1 in the microgravity environment, and the thermocapillary effect dominates the convection process. Carefully designed experiments on the floating half zone for measurement of the critical Marangoni number were completed by using a drop shaft facility with $10 \mathrm{~s}$ of microgravity period in the JAMIC of Japan, and fruitful results were obtained [36,37]. The typical scale of a liquid bridge is still small because the applied temperature difference cannot be adjusted during the limited microgravity period. Several experiments of larger typical scale of the liquid bridge were performed on board the microgravity sounding rocket TEXUS with 6 min of microgravity period, and the critical Marangoni number was obtained during an increasing applied temperature difference [38-40]. Most important experiments on thermocapillary convection in a floating half zone were performed by Monti and co-workers [41,42] on board the Spacelab of the D2 mission $[41,42]$, and the experimental results were summarized in Table 3. It could be seen that the critical Marangoni number is larger in one order of magnitude in comparison with that of the 
Table 3 Experimental results of the D2 mission on board Spacelab

\begin{tabular}{cccccc}
\hline \hline Diameter $(\mathrm{mm})$ & Height $(\mathrm{mm})$ & $A$ & $\Delta T\left({ }^{\circ} \mathrm{C}\right)$ & $(\mathrm{Ma})_{c}$ & $f_{c}(\mathrm{~Hz})$ \\
\hline 30.0 & 30.0 & 1.0 & 10.1 & 68,000 & $0.25 \pm 0.05$ \\
30.0 & 45.0 & 1.5 & 17.9 & 180,000 & $0.25 \pm 0.05$ \\
30.0 & 60.0 & 2.0 & 15.9 & 213,000 & $0.32 \pm 0.02$ \\
45.0 & 33.75 & 0.75 & 15.2 & 114,000 & $0.20 \pm 0.02$ \\
45.0 & 45.0 & 1.0 & 12.0 & 120,000 & $0.14 \pm 0.02$ \\
45.0 & 67.5 & 1.5 & 12.3 & 185,000 & $0.18 \pm 0.02$ \\
60.0 & 45.0 & 0.75 & 16.5 & 166,000 & $0.12 \pm 0.02$ \\
60.0 & 60.0 & 1.0 & 17.2 & 230,000 & $0.12 \pm 0.02$ \\
\hline \hline
\end{tabular}

experiments on the ground, and the critical Marangoni numbers are increased with the increasing diameters of the cylindrical liquid bridges for the same aspect ratio $A$. This means that the dimensional scale is also an important parameter for the onset of oscillation even for cases of liquid bridges with larger scales in the microgravity environment. Furthermore, the space experimental results turned out to be contrary to many experiments on the ground. The critical Marangoni number decreases during the increase of the aspect ratio on the ground but increases with the increase of the aspect ratio in space experiments for a fixed diameter of the liquid bridge. It seems that more space experimental results are necessary. At least two space missions will be on board the Russia and the Japanese modules of the International Space Station, respectively, in the near future, and more data will be obtained.

To search the sensitive critical parameter is important in understanding the mechanism of thermocapillary convection. The aspect ratio is usually used to describe the geometric feature, and the liquid bridge is assumed to be a cylindrical shape in most experimental models on the ground. In fact, the ratio liquid bridge volume $V / V_{0}$ is another geometrical critical parameter in addition to the aspect ratio, and is defined as

$$
V / V_{0}=\frac{1}{r_{0}^{2}} \int_{0}^{1} r^{2}(z) d z
$$

where $r=r(z)$ is the equation of the free surface of a cylindrical liquid bridge. This new geometrical parameter is more sensitive to the onset of oscillation in comparison with the aspect ratio. The critical Marangoni number depending on the ratio of the liquid bridge volume has been experimentally studied such as in Refs. $[28,16,17,43,44]$ and summarized in a book [13]. The margin instability curves separated into two branches of the slender liquid bridge and the fat liquid bridge are given typically in Fig. 3 . These results imply that the curvature variation of the free surface determined by the stress balance in the normal direction is important in the thermocapillary convection, which is driven by the stress balance in the tangential direction. It needs a careful comparison of the experimental results for a liquid bridge of small scale on the ground and the theoretical results for a cylindrical liquid bridge in the microgravity environment even in the case of $V / V_{0}=1$ because of the curvature effect of the free surface.

It is important to understand the essentials of thermocapillary convection to get more detailed information on the physical fields and their interactions in the liquid bridge and to develop new diagnostic techniques and experimental methods. One of the progresses on the experimental system is to introduce a transparent material, such as a sapphire or a glass disk, to replace one of the metal rods in the floating half zone system, firstly by Hirata et al. [37] and Frank and Schwabe [45], as shown in Fig. 4. In this case, the flow field in a horizontal cross section could be observed in addition to the one in a vertical cross section, and the flow fields and flow patterns related to different azimuthal modes $m$ could be observed. The experimental results of the flow fields in both the horizontal and vertical cross sections discover the fea- tures of three-dimensional velocity fields in some sense, and are beneficial to compare with the results of theoretical analyses. The experimental results of a floating half zone system consisting of a transparent disk and a metal rod are compared experimentally with the one consisting of two metal rods, and the flow fields in the horizontal direction are observed especially [46].

The waves in fluids are an interesting subject in the fluid mechanics and have been extensively studied. The microgravity environment supports an excellent opportunity to study the waves in fluids dominated by the thermocapillary effect, and this is a new sort of free surface waves. Hu et al. [47] developed an optical microvideo measurement system to observe the variation of liquid bridge radii, and the measurements directly gave the oscillation of free surface in a vertical cross section of the liquid bridge. The results show that the amplitude of free surface oscillation is of a few micrometers in a liquid bridge of a few millimeters in diameter, and the onset of free surface oscillation is a bit earlier than the onset of temperature oscillation, as shown in Fig. 5 [48]. Furthermore, Shu et al. [49] developed an optical diagnostic method to observe the three-dimensional free surface waves by measuring the distortion of a laser beam that passed through a grid mask reflected from the free surface. The distorted grid pattern recorded by a charge coupled device (CCD) camera was reversed in real time to give the evolution of free surface deformation and oscillation, as shown in Fig. 5. Both optical diagnostic methods were involved in the microgravity experiments to measure the free surface oscillations in a small liquid bridge by using the drop shaft facility in JAMIC of Japan [50].

The experimental studies are essential for the study of the ther-

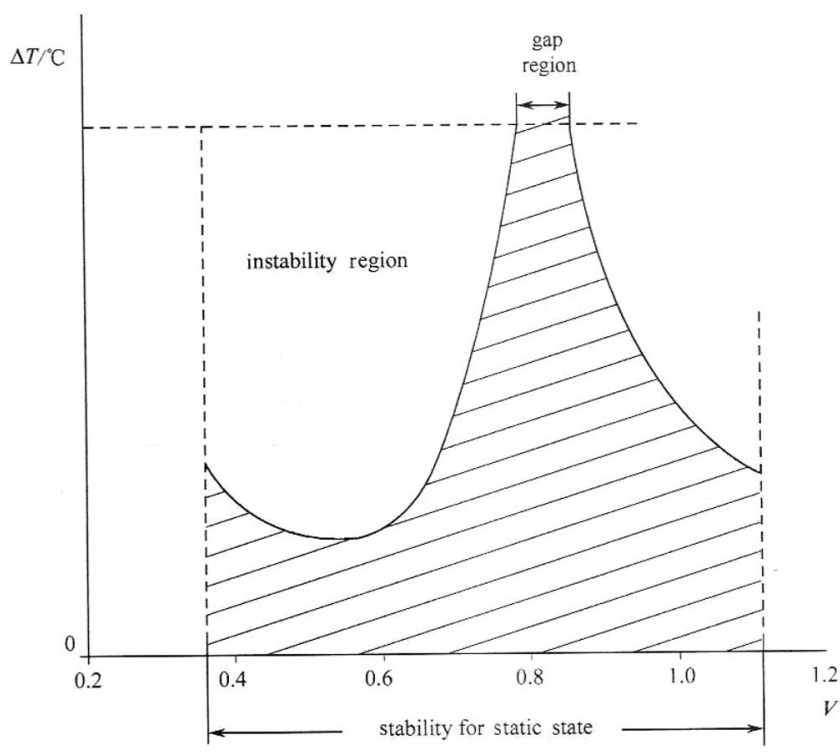

Fig. 3 The critical Marangoni number depending on the volume of liquid bridge 


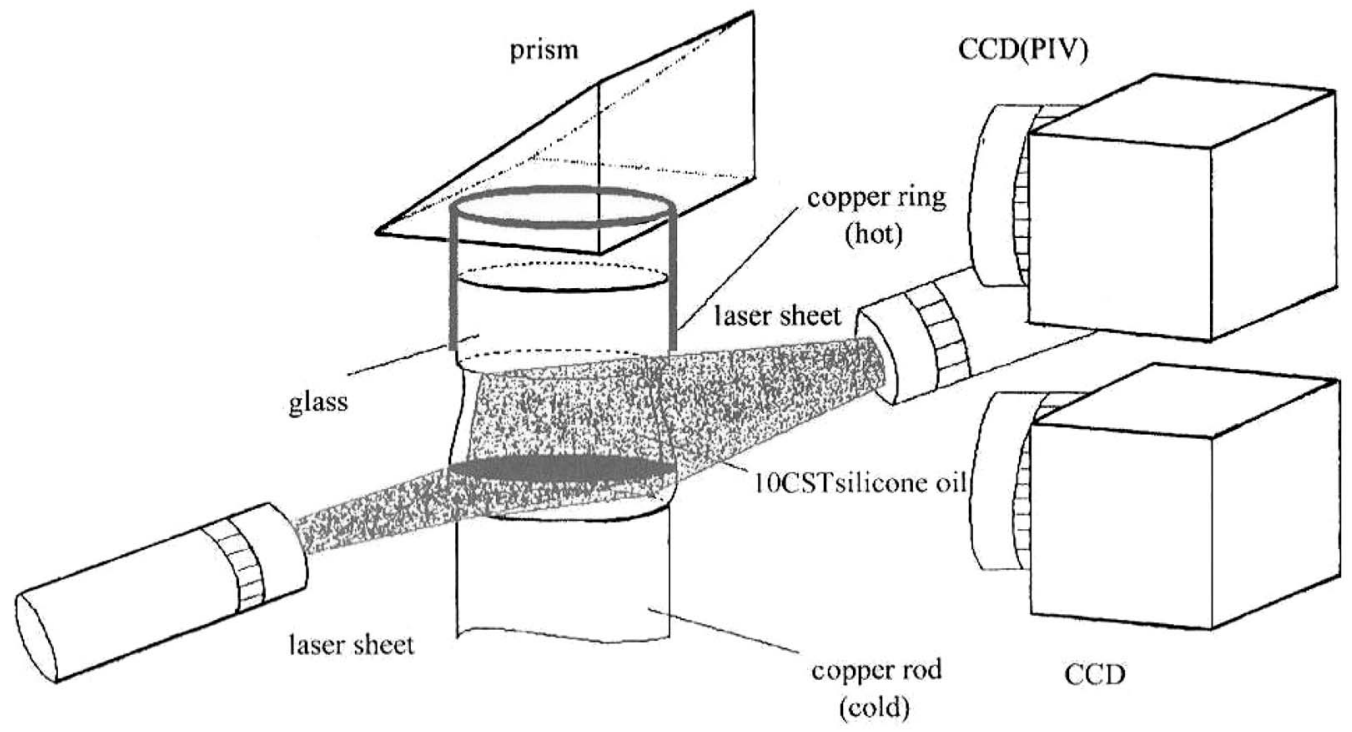

Fig. 4 The PIV measurement system in two cross sections of a liquid bridge

mocapillary convection. In summary of the experimental studies, we can measure the temperature distributions in the liquid bridge at fixed positions and near the free surface by the thermocouples, the flow fields in both a vertical cross section and a horizontal cross section by the particle tracking velocimetry (PTV) method, and free surface variations and surface waves (Fig. 6) in a view field of a few millimeters square by the optical diagnostic methods. There, fields may be written in two parts:

$$
\mathbf{V}=\mathbf{V}_{0}+\mathbf{V}^{\prime} \quad T=T_{0}+T^{\prime} \quad R=R_{0}(z)+R^{\prime}
$$

where subscript 0 denotes the average part of slow variation and the prime denotes the fluctuation part of oscillation. The experimental results of oscillatory thermocapillary convection give the relationships in the order of magnitude as

$$
\left|\frac{V^{\prime}}{V_{0}}\right|=O\left(10^{0}\right) \quad\left|\frac{T^{\prime}}{T_{0}}\right|=O\left(10^{-1}\right)-O\left(10^{-2}\right) \quad\left|\frac{R^{\prime}}{R_{0}}\right|=O\left(10^{-3}\right)
$$

According to relationship (10), the fluctuation velocity has the same order of magnitude as the average velocity. It means that the onset of thermocapillary oscillation is a strong nonlinear process in principle, and the analyses of linear perturbation instability used extensively in studying the onset process is probably a bit

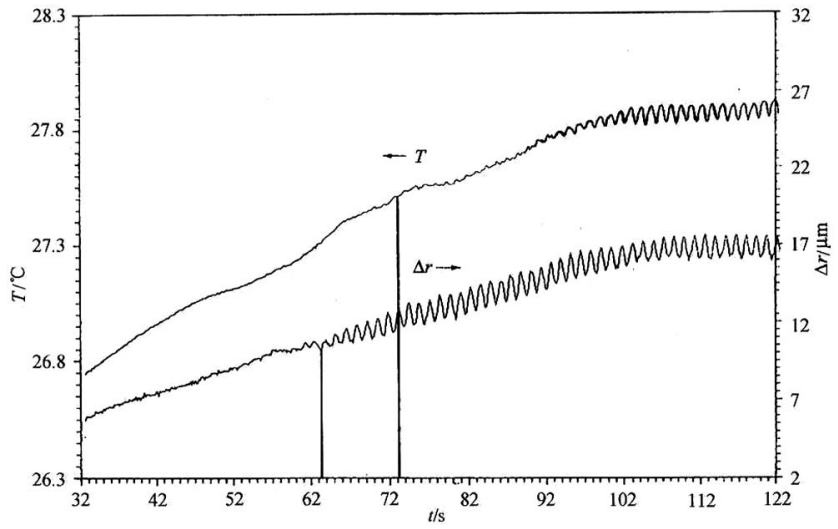

Fig. 5 The onset of oscillatory processes on the coordinated measurements of temperature and free surface radii during a heating rate $0.14^{\circ} \mathrm{C} / \mathrm{s}$ in a liquid bridge too simple for the study of the nonlinear behavior. The results on the free surface in Eq. (10) show that the deformation of the free surface is very small, and the energy associated with the free surface variation is not enough to excite the velocity and temperature fluctuations, which may be the sources to induce the free surface fluctuation. Due to the results of relationship (10), the average and fluctuation parts of the enthalpy and the kinetic energy may be given in order of magnitude as

$$
\begin{array}{cl}
O\left(\rho C_{\rho} T_{0}\right)=10^{8}-10^{9} \mathrm{~g} / \mathrm{cm} \mathrm{s}^{2} & O\left(\rho C_{\rho} T^{\prime}\right)=10^{6}-10^{8} \mathrm{~g} / \mathrm{cm} \mathrm{s}^{2} \\
O\left(\rho V_{0}^{2} / 2\right)<10^{2} \mathrm{~g} / \mathrm{cm} \mathrm{s}^{2} & O\left(\rho V_{0} V^{\prime}\right)<10^{2} \mathrm{~g} / \mathrm{cm} \mathrm{s}^{2}
\end{array}
$$

These results imply that the temperature fluctuation cannot be excited by the kinetic energy, and the main source for the onset of oscillatory thermocapillary convection should be the terms related to the thermal energy, as discussed in detail in Ref. [51].

Recently, the studies are devoted to find the process of two bifurcation transitions for the onset of oscillation in a floating half zone of large Prandtl number fluids, that is, the steady and axial symmetric convection transits at first to the steady and axial nonsymmetric convection (with usually azimuthal mode $m=1$ ), and then to the oscillatory thermocapillary convection (with usually azimuthal mode $m=2$ ) during the increase of the applied temperature difference in a fat liquid bridge [52-54]. This conclusion is similar to the transition process in a floating half zone of small Prandtl number fluids, where two critical Marangoni numbers related to the two bifurcation transitions are discussed [14]. The process of two bifurcation transitions is an interesting subject, especially associated with the mechanism of the onset of oscillatory thermocapillary convection. The so-called hydrothermal instability has been broadly used to explain the onset of oscillatory thermocapillary convection, and the hydrothermal instability requires a traveling wave as the oscillatory mode. It is obvious that the first bifurcation transits from a steady state to another steady state, and there has no traveling wave during the transition. The second bifurcation transition may probably relate to the mechanism of hydrothermal instability, and in this case, the transition from the axial nonsymmetric convection to the oscillatory thermocapillary convection should be analyzed. It seems that the conclusion on two bifurcation transitions needs to be explained by a mechanism that is probably different from the hydrothermal instability. 

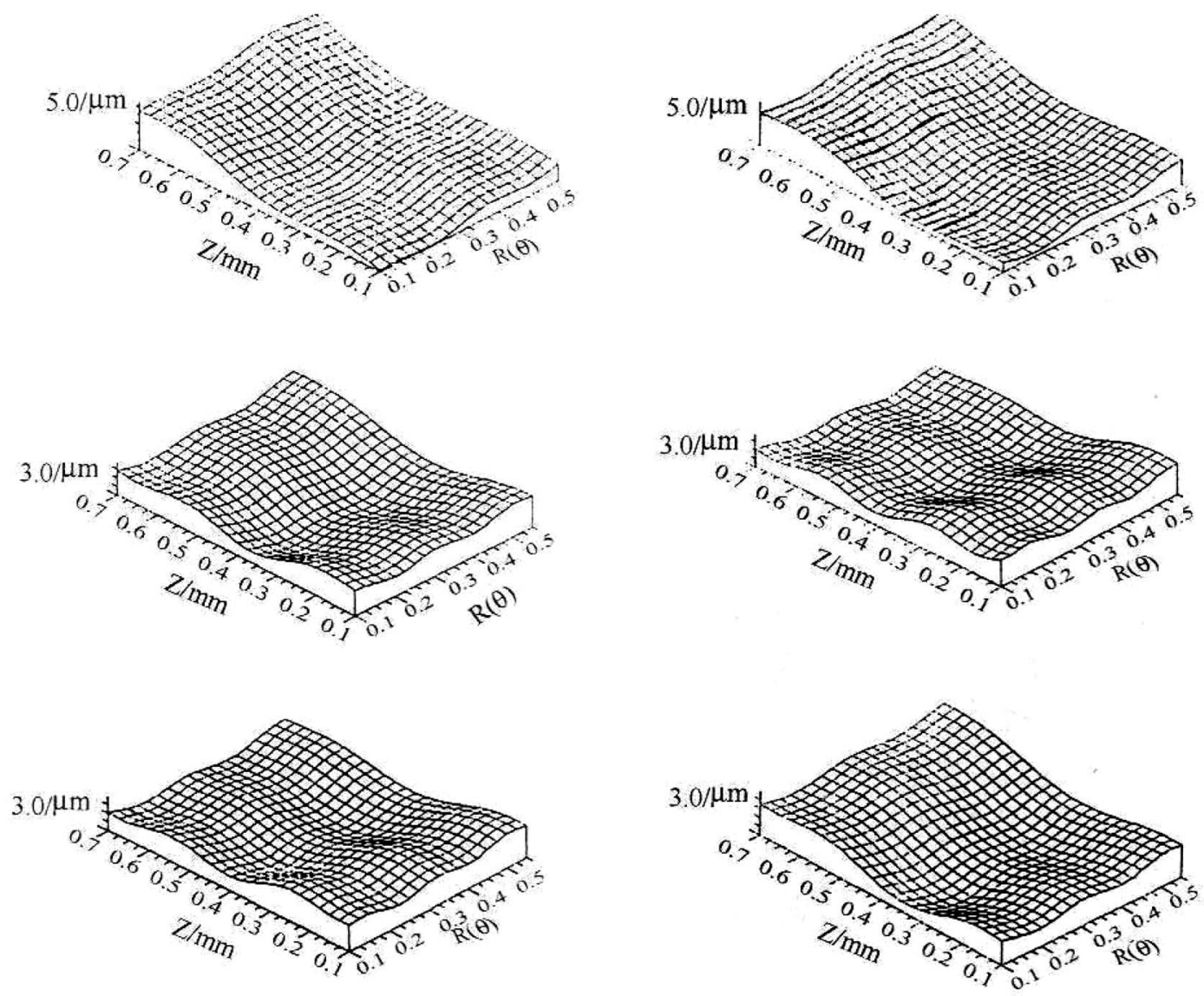

Fig. 6 The measurement results of free surface waves of oscillatory thermocapillary convection in a liquid bridge of floating half zone

\section{Theoretical Models on the Onset of Oscillation}

The method of linear instability analysis deals with the small deviations from a basic state and has been used to investigate the onset of oscillatory thermocapillary convection in a liquid bridge of floating half zone. The basic state of a laminar thermocapillary convections was simplified as a linear flow or returning flow in a liquid layer or bridge of infinite extension, a free surface with constant temperature gradient [55-57]. In the recent years, many studies have been devoted to the use of the method of linear instability analysis, and the axial symmetric solution of NavierStokes equations of a given aspect ratio is solved as the basic state for a cylindrical liquid bridge [58-62] and a curved liquid bridge [63-65]. The eigenvalue problem of the perturbation state analyzed for a given integer azimuthal wave number $m$ is solved in order to find the marginal instability curve with zero growth rate $\omega_{r}$, where the eigenvalue $\omega=\omega_{r}+\omega_{i}$, composed of growth rate $\omega_{r}$ and oscillatory frequency $\omega_{i}$. It is noted that the results of linear instability analysis describe the very beginning of the transition from the laminar to the oscillatory convection, but do not certainly describe the behavior of the nonlinear process of large deviation from the laminar convection, such as the floating half zone convection in general.

The energy theory stability is also applied to investigate the onset of oscillatory thermocapillary convection and to prove a criterion guaranteeing stability in the floating half zone of finite aspect ratio $[66,67]$. The energy stability theory is also a linear analysis of the perturbation state that deviated from a basic state. The stability criterion of energy theory stability is obtained by the analysis of perturbation "total energy," but not by the calculation of an eigenvalue problem as in the method of linear instability analysis. Therefore, the results of energy theory instability give the sufficient condition of stability to disturbances of small amplitudes. The equations for a basic state perturbation state may be obtained in the same way in the method of linear stability analysis. By taking the inner product of the perturbation momentum equation with perturbation velocity $\mathbf{v}^{\prime}$ and multiplying the perturbation energy equation by $\lambda \operatorname{Pr} T^{\prime}$, the equation for perturbation total energy $E=1 / 2 \int\left(\overline{v^{\prime}} \cdot \overline{v^{\prime}}+\lambda \operatorname{Pr} T^{\prime 2}\right) d V$ is demonstrated by adding both equations integrated over the liquid bridge volume, that is,

$$
\frac{1}{E} \frac{d E}{d t}=\frac{1}{E}(-\operatorname{Pr} D-\mathrm{Ma} I+\operatorname{Pr} J)
$$

where $\lambda$ is a positive coupling parameter, and $D$ and $I$ are, respectively, the related volume integrations of perturbation terms, and J is the integration on the free surface [66]. The Marangoni number is adopted as the instability parameter, and the other parameters related to the problem are fixed. The critical Marangoni may be obtained by the maximum problem of Eq. (11), and then the critical Marangoni number is given by

$$
(\mathrm{Ma}) c=\max _{\lambda>0} \operatorname{Ma}(\lambda)
$$

The critical Marangoni number was obtained for different aspect ratios and Prandtl numbers. The results gave $\mathrm{Ma}=6274$ for a twodimensional perturbation analysis [66], and $\mathrm{Ma}=6254,1503$, 2633, and 3545 associated with perturbation azimuthal mode $m$ $=0,1,2$, and 3 for a $3 \mathrm{D}$ perturbation analysis [67] in the case of $\operatorname{Pr}=1, A=1$ and $\mathrm{Bi}=0.3$. The results of energy theory instability are compared with the linear instability analyses, and both results are comparable. 
Several theoretical models on mechanisms will be discussed in the following subsections.

4.1 Mechanism of Hydrothermal Instability. Smith and Davis suggested the hydrothermal instability to explain the onset of oscillatory thermocapillary convection in a liquid layer of infinite extension by the method of linear instability analysis [55] and in a cylindrical liquid bridge of infinite extension under the microgravity environment [57]. The quantities of the perturbation state were assumed in proportion to $\exp [i(\alpha x+\beta y-\omega t)]$, where the coordinates $(x, y)$ are in the cross section of the liquid layer or liquid bridge. Then, the 3D disturbed equations may be solved numerically to determine the critical Marangoni number $\mathrm{Ma}_{c}$. For the basic state of returning flow,

$$
\frac{u}{u^{*}}=\frac{3}{4}\left(\frac{z}{d}\right)^{2}-\frac{1}{2}\left(\frac{z}{d}\right) \quad v=w=0
$$

the results gave a traveling wave associated with the onset of oscillation, and the wave was defined as the hydrothermal wave or named the instability as the hydrothermal instability, satisfied for the entire range of the Prandtl number Pr. The mechanism of hydrothermal instability is associated with a balance between the heat convection and heat conduction, and the eigenvalue of the system can be solved from the energy equation $[68,69]$.

The critical Marangoni number given by such a simplified model is small in the order of magnitude in comparison with the one given by the experiments in the liquid bridge of finite extension. The temperature gradient on the free surface is constant in the analysis of hydrothermal instability for the liquid layer or liquid bridge with infinite extension, and is quite nonuniform in a liquid layer or liquid bridge with finite extension. The idea of hydrothermal instability has been used broadly to explain the onset of oscillatory thermocapillary convection.

4.2 Mechanism of Hydrodynamic Instability. The hydrothermal instability was quested firstly for the case of small Prandtl numbers. Levenstam and Amberg (1995) analyzed a liquid bridge of small Prandtl number $\operatorname{Pr}=0.01$ by direct numerical simulation and found that the axisymmetric laminar thermocapillary convection transited firstly to a steady nonaxisymmetric state and then to an oscillatory state [18]. This result implies that there are two bifurcation transitions related to two critical Marangoni numbers, which are $(\mathrm{Ma})_{c 1}=1960$ and $(\mathrm{Ma})_{c 2}=6250$ for a liquid bridge with aspect ratio $A=1$. These transition processes are explained by a mechanism of hydrodynamic instability and are obviously different from the hydrothermal wave, which is associated with a transition directly to an unsteady state of the traveling wave. The onset of oscillatory thermocapillary convection is usually explained by the mechanism of hydrodynamic instability for small Prandtl liquid bridges.

A region of intermediate Prandtl numbers will separate the flow characteristics of the hydrodynamic instability in cases of small Prandtl number liquid bridges and the hydrothermal instability in cases of large Prandtl number liquid bridges. The critical branches for a cylindrical liquid bridge of $A=1$ are divided into steady convection $m=2$ for $0 \leqslant \operatorname{Pr}<0.057$, oscillatory convection $m=3$ for $0.057 \leqslant \operatorname{Pr}<0.070$, standing wave of oscillatory convection $m=2$ for $0.070 \leqslant \operatorname{Pr}<0.183$, standing wave of oscillatory wave $m=3$ and $m=4$ for $0.183 \leqslant \operatorname{Pr}<0.840$, and traveling wave of oscillatory convection $m=2$ for $\operatorname{Pr} \geqslant 0.840$ [14]. In principle, the linear instability analysis can give only the onset of first bifurcation, and the first bifurcation is not proven by the experiment because of the difficulty of notransparency and surface pollution for noncontact diagnostic in a liquid bridge of small Prandtl numbers. The unsteady and 3D numerical simulation may describe the complete evolution process with both bifurcation transitions.

Recent studies show that two bifurcation transitions, similar to the cases of small Prandtl number liquid bridges, exist also in the case of large Prandtl number liquid bridges with larger ratio liquid bridge volume and smaller aspect ratio, and the results are obtained by the unsteady $3 \mathrm{D}$ numerical simulation $[52,53]$. The advantage of using a liquid bridge of a larger Prandtl number is easy for experimental diagnostics, and the evolution process of two bifurcation transitions are performed experimentally in a liquid bridge of $10 \mathrm{cS}$ silicon oil, as discussed in the previous section $[52,54]$. The experimental results are comparable with the results of numerical simulation.

4.3 Mechanism Based on Free Surface Deformation. Kamotani and Ostrach suggested a mechanism based on the free surface deformation (see, for example, Refs. [70,71]). Because the critical Marangoni number depends on the typical scale $l$, as shown in relationship (7), they introduced a new aspect and parameter to describe the free surface deformation. According to this mechanism, the hot fluid is originated in the heated region (near the corner for a liquid bridge or near the center for a cylindrical container), which is relatively small, and the velocity in the small heated region is relatively larger. The typical flow time in this region is small relative to the overall convection time. The smaller flow time is associated with the very quick change of the temperature field and, thus, with the thermocapillary drive force; consequently, the return flow of overall convection cannot respond immediately to the quick change of the surface flow in the heated region, and the free surface will deform first to generate sufficient pressure gradient for the response. In other words, there exists a time lag, which is the primary factor in the oscillation mechanism.

Based on the order of magnitude analysis, a new parameter named $S$ is introduced as follows:

$$
S=\left(\frac{\rho \kappa}{\sigma \ell}\right)^{1 / 2} \operatorname{Ma}^{11 / 8} \operatorname{Pr}^{1 / 8}\left(\frac{\mu_{c}}{\mu_{m}}\right)^{1 / 8}
$$

where the subscripts $c$ and $m$ imply, respectively, the value at low temperature $T_{c}$ and mean temperature $\left(T_{H}+T_{c}\right) / 2$. The experimental results gave the margin condition $S=69 \pm 14 \%$ for the onset of oscillatory thermocapillary, or the thermocapillary convection becomes oscillation if the $S$ parameter is roughly larger than 70. In this case, the critical Marangoni number is given as

$$
(\mathrm{Ma})_{c}=21.973 \operatorname{Pr}^{-1 / 11}\left(\frac{\mu_{m}}{\mu_{c}}\right)^{1 / 11}\left(\frac{\sigma \ell}{\rho \kappa}\right)^{4 / 11}
$$

The space experiments have been devoted to checking this theoretical model for the thermocapillaty convection in an open cylindrical container with a free surface heated by a $\mathrm{CO}_{2}$ laser near the center region $[71,72]$. However, the mechanism is applied also to the floating zone convection [35].

It is noted that this mechanism requires a small heated region, such as a heater region, in the open container or the corner region of a floating half zone, and cannot be applied to a liquid layer or liquid bridge of infinite extension with a constant temperature gradient on the free surface, as discussed in the hydrothermal instability [55]. The idea of this mechanism is different from the one of hydrothermal instability.

The experimental results on the ground $[47,48]$ and in the microgravity environment $[49,71,72]$ show that the deviation scale of the free surface during the oscillation is as small as $O\left(10^{-3}\right)$ in order of magnitude in comparison with the typical scale of the system, as shown in relationship (10). This means that the energy related to the free surface deformation is relatively small.

4.4 Unsteady and Three-Dimensional Numerical Simulation. Unsteady and three-dimensional numerical simulations are excellent methods to study the complete transition process of the floating zone convection, especially for the strong nonlinear behavior. The unsteady and two-dimensional numerical simulation have been used to study the transition process since the 1980s; however, the detailed features, such as the 3D features of experimental results, can be shown clearly by the unsteady and 
three-dimensional numerical simulation.

Rupp et al. (1989) developed an unsteady and 3D numerical simulation method to compare the results of numerical simulation with the experimental results on the onset of oscillatory thermocapillary convection in a floating zone of small Prandtl number fluids [24]. Furthermore, Levenstam and Amberg (1995) discovered the two bifurcation transitions in the floating half zone of small Prandtl number fluid $\operatorname{Pr}=0.01$ [18]. Afterward, many numerical studies have been devoted to investigate the bifurcation characteristics in the floating half zone of small Prandtl number fluids. Recently, a benchmark research is devoted jointly by nine groups to compare their results for the floating half zones of small Prandtl number fluids with curved free surface, and the deviation of the results is reasonable in a range of $15 \%$ [73].

The unsteady and 3D numerical simulation method has been devoted to the study of the floating half zone with a cylindrical configuration $[74,75]$ and with a curved free surface [76] of larger Prandtl number fluids. This method is also used to study the conditions of two bifurcation transitions in the liquid bridge of large Prandtl numbers $[52,53,77]$. These numerical results of larger Prandtl number fluids are advantageous in comparing with the three-dimensional evolution characteristics of the experimental results, which are strongly nonlinear.

4.5 Comparison With Experiments. Most thermocapillary experiments of floating half zone convection have been performed on the ground with a typical scale of few millimeters in diameter for avoiding the buoyancy effect in comparison with the thermocapillary effect. The simplified theoretical model of hydrothermal instability model gave a lower critical Marangoni number in order of magnitude in comparison with the experiment [57] because the theoretical model emphasized the essential and did not even consider the influence of the Prandtl number. Recent results of unsteady and three-dimensional numerical simulation gave the critical Marangoni number and flow pattern [53], which agree reasonably with the experimental results on the ground in a floating half zone of small diameter, for example, $10 \mathrm{cS}$ silicon oil [54]. Many factors will induce the influences on the results, and the comparison needs to be more careful for the floating half zone of higher liquid temperature in small scale.

The unsteady and 3D numerical simulation has been devoted also to the study of the onset of oscillation in the zero gravity condition, and has been compared with the results of space experiments on board the Maxus 1B and Spacelab D-2 on the cylindrical floating half zone with a diameter of several centimeter [71]. The numerical results of critical Marangoni numbers were respectively $6.4 \times 10^{4}$ for the Maxus mission and $1.9 \times 10^{5}$ for the D2 mission, and the space experimental results of the silicon oil liquid bridges were $6.5 \times 10^{4}$ and $1.8 \times 10^{5}$, respectively. The results showed also that all the flow patterns are related to mode $m=1$. It seems that the unsteady and $3 \mathrm{D}$ numerical simulation can give the results that agree reasonably with the space experimental results of floating half zone of silicon oil.

The discovery of the second bifurcation transition in the floating half zone convection gave the critical Reynolds number $(\mathrm{Re})_{c 2}=6250$ for $A=1$ and $\operatorname{Pr}=0.01$ [18], and the related second critical Marangoni number is $(\mathrm{Ma})_{c 2}=62.50$. Many theoretical results gave discussions on the first critical Marangoni number but were limited on the second critical Marangoni number [73], which can only be compared with the experiment. Experimental results on the second critical Marangoni numbers were 43.3 for $A=2.2$ and $\operatorname{Pr}=0.012-0.008$ (related to temperature $T=570-770 \mathrm{~K}$ ) [31], 194 for $A=2$ and the same Prandtl number [30], and less than 900 for $A=0.6$ and $\operatorname{Pr}=0.025[26,27]$. Based on the experimental and theoretical results of medium Prandtl numbers, the critical Marangoni number is decreased during the increase of the aspect ratio. Therefore, the theoretical result of the second Marangoni number in a fluid of $\operatorname{Pr}=1$ in the case of $A=2.2$ will be less than 62.50. To compare with the Japanese experiment [31] in detail, the second critical Marangoni number given by numerical simulation is $(\mathrm{Ma})_{c 2}=37.48$ for $\operatorname{Pr}=0.009$ and $A=2.2$ [78]. The theoretical results predicted a smaller second critical Marangoni number, smaller oscillatory amplitude, and higher critical frequency in comparison with the experimental results. Further numerical simulation gave the critical conditions of $(\mathrm{Ma})_{c 2}=29$ and $f_{c}=1.9 \mathrm{~Hz}[79,80]$, which still do not agree with the experimental results of $(\mathrm{Ma})_{c 2}=43.3$ and $f_{c}=0.08 \mathrm{~Hz}$ in a quantitative sense, but both the theoretical and experimental second critical Marangoni numbers agree in a reasonable range.

It is noted that the free surface pollution is a serious problem to change the surface tension in cases of higher temperature experiment, and the deviation of free surface configuration from a cylinder can induce a large difference of critical Marangoni number, especially in comparison of the theoretical result with the experimental result. These subjects need to be studied in detail in the future.

\section{Transition to Turbulence}

When the applied temperature difference continuously in creases after the onset of thermocapillary oscillatory convection, the fluctuation physical quantities become obviously more nonlinear in their behavior, and eventually the oscillatory convection will transit to turbulence or chaos if the temperature difference is large enough. The chaos is a general behavior of the nonlinear system in nature, and the system of thermocapillary convection supports a new area for studying the nonlinear sciences. The transition process, such as the chaos behavior, has been extensively investigated for the Benard convection, and the route to turbulence is a typical subject in the fluid mechanics. The experiments on the chaos behavior of the thermal convection have been studied extensively, and the routes to turbulent convection depend complicatedly on many parameters, such as the geometric parameters (such as the aspect ratio, the diameter of the liquid bridge, the liquid bridge volume), the Prandtl number, etc.

A route of period doubling bifurcation to chaos was demonstrated theoretically by Feigenbaum in 1979 [81], and the subharmonic bifurcation satisfies the universal relations

$$
\delta_{n}=\frac{f_{n+1}-f_{n}}{f_{n+2}-f_{n+1}} \rightarrow 4.6642016, \quad n \rightarrow \infty
$$

where subharmonic bifurcation of $f_{n}=f_{0} / 2^{n}(n=2,3,4, \ldots)$ appears successively during the increasing temperature difference and $f_{0}$ is the main frequency.

The turbulence developing from the oscillatory thermocapillary convection in a liquid bridge of floating half zone was discussed by Chun in 1984 [82]. Probably due to the difficulty, the subject has not been discussed in a decade afterward. Both theoretical and experimental studies were devoted to the search of the subharmonic bifurcation in a floating half zone system [83]. A liquid bridge of $10 \mathrm{cS}$ silicon oil $(\operatorname{Pr}=105.6)$ with $d_{0}=3 \mathrm{~mm}, A=0.95$, and $d_{\min } / d_{0}=0.7$ was used, and $d_{\min }$ is the minimum diameter of the liquid bridge. The diameter ratio $d_{\min } / d_{0}$ is related uniquely to the ratio volume $V / V_{0}$. The frequency spectra of surface temperature at a fixed point were given by numerical simulation, as shown in Fig. 7, where the subharmonic sequence clearly appears. The numerical results gave $\delta_{4}=4.853$, which was closed comparable to the universal constant 4.664 given by Feigenbaum [81]. Recently, an experiment gave the subharmonic sequence in a liquid bridge of a floating half zone system with $d_{0}=4 \mathrm{~mm}, A=0.96$, and $d_{m} / d_{0}=0.9$, and the results show that $\delta_{2}=4.69 \pm 0.05$ and $\delta_{4}$ $=4.6 \pm 0.1$, as shown in Fig. 8 [84].

Frank and Schwabe continued the studies on the subject by experiments on the ground [45] and on board the Gas Away Special (GAS) payload G-141 of STS 89, and the space experimental period was $14 \mathrm{~h}$ [85]. The experimental results gave some transitionary temporal phenomena accompanying the system's way to chaotic behavior, especially the observations of the transition to 


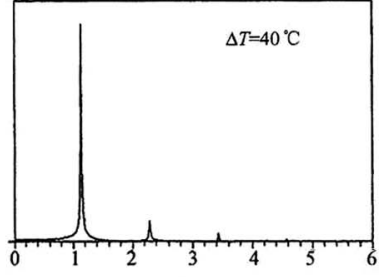

(a) $f / \mathrm{Hz}$

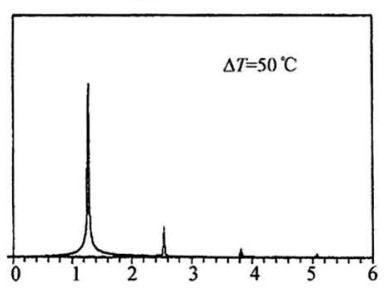

(b) $\mathrm{f} / \mathrm{Hz}$

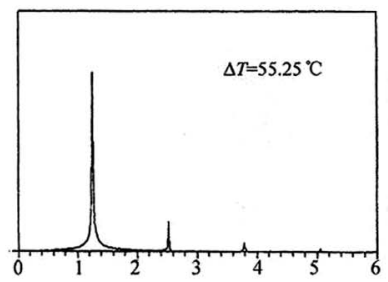

(c) $f / \mathrm{Hz}$

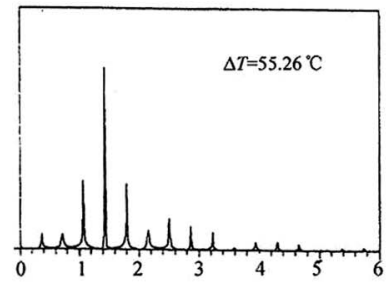

(d) $f / \mathrm{Hz}$

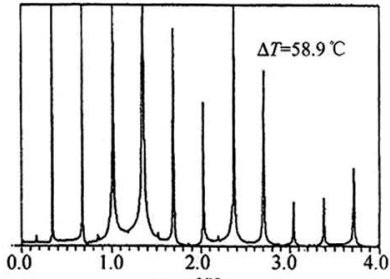

(e) $f / \mathrm{Hz}$

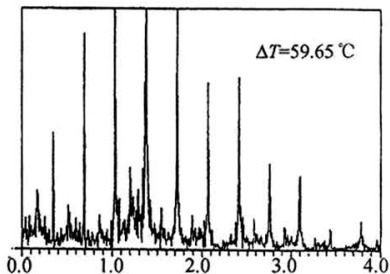

(f) $f / \mathrm{Hz}$

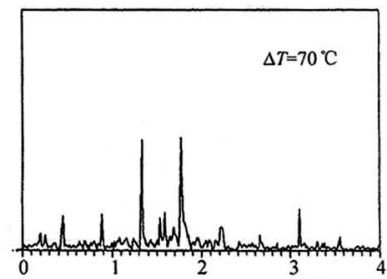

(g) $f / H z$

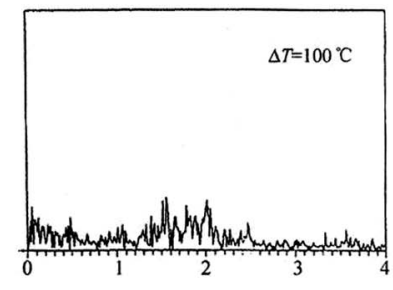

(h) $f / \mathrm{Hz}$
Fig. 7 The spectrum of surface temperature at $z=0.16 \mathrm{~mm}$ given by a numerical simulation result

chaotic thermocapillary flow and various stages on the route to chaos. The experiments were performed in the liquid bridges of larger Prandtl number fluids $\operatorname{Pr}=7,49$, and 65 on the ground, and $\operatorname{Pr}=7$ on board STS 89. The diameter and length of the liquid bridge are small in millimeters in order of magnitude. The applied temperature difference and the aspect ratio are the main parameters to be considered for the transition process.

As a new subject of the thermocapillary convection in the microgravity environment, the studies on transition to turbulence are preliminary at the present time. We have only limited knowledge on this subject. We need to study the dependence of different routes on the different parameters, and then to discover more examples of the evolutions of routes in detail. The subject is attractive in the frontier of microgravity science and also in the nonlinear science.

\section{Some Interesting Recent Research}

An overview of the floating zone convection is given in the previous sections, and the history and the main progress related especially to the mechanism studies are summarized. Space experiments on the floating half zone of larger Prandtl number fluids are arranged to be on board the International Space Station in the near future, and the results of these experiments may give important contributions to understand the essentials of floating zone convection. Recently, there are some interesting research subjects, which will be discussed as follows.
6.1 Effect of Heat Loss From Free Surface. Recent detailed studies of floating zone convection in the liquid bridge of medium and large Prandtl number fluids show that the heat loss has obvious influence on the critical Marangoni number, although the idea had been generally discussed earlier. Most theoretical studies on the floating zone convection have assumed a zero heat loss from the free surface, that is, the zero Biot number $\mathrm{Bi}=0$. The early numerical simulations conclude that the effect of heat loss can stabilize the convection. However, the recent experiment of high Prandtl number fluid shows that the critical Marangoni number is decreased for the increasing Biot number [86]. In addition to the temperature difference, the temperature of cold rod and the temperature of ambient gas are two more dimensional critical parameters, and they change the critical Marangoni number even when the temperature difference are the same [87].

Results of detailed calculations agree with the experimental results in liquid bridge $2 \mathrm{cS}$ silicon oil $(\operatorname{Pr}=28.1)$, and the critical Marangoni number is decreased with the increasing Biot number; on the other hand, the critical Marangoni number is increased with the increasing Biot number in the case of the acetone liquid bridge of medium Prandtl number fluid $\operatorname{Pr}=4.38$ [88]. Numerical simulation is also devoted to a case of Prandtl number $\operatorname{Pr}=14$, and the critical Marangoni is linearly decreased when the Biot is increased in the range $0 \leqslant \mathrm{Bi} \leqslant 1.8$; however, the critical Marangoni number in the case of $\mathrm{Bi}=9.0$ is larger than the one in the case of $\mathrm{Bi}=0$ [89].

Both the results of theoretical and experimental studies gave the agreeable conclusion in cases of large Prandtl number fluids; however, the dependent rule is different in the case of medium Prandtl number fluids. The floating zone convection has abnormal characteristics in the medium Prandtl number fluids, and it needs to be studied in the future.

6.2 Encapsulated Floating Half Zone. The onset of oscillatory convection in a floating zone is associated with the appearance of striation in the crystal during the crystal growth, and avoiding or delaying the onset of oscillation is important in the floating zone technique. One of the ideas is an encapsulated floating zone, in which the floating liquid zone is encapsulated by an immiscible liquid column for controlling the floating zone convection by heat transfer and momentum equilibrium at the interface of two immiscible liquids [90]. This is a bit similar to the heat loss from the free surface between liquid and gas in pure floating zone convection in some sense.

An experiment was completed, and two immiscible liquids are adopted as silicon oil KF-96 and Fluorinert FC-70 [91]. In the case of KF-96 of the inner column and FC-70 of the outer column, the critical Marangoni number of inner floating half zone is obviously larger than that without the encapsulated FC-70 column. In the case of the FC-70 inner column and the KF-96 outer column, the critical Marangoni number is larger than that of a KF-96 floating zone; however, it is obviously smaller than that of the FC-70 floating half zone without the encapsulated KF-96 column. The results mean that the onset of oscillatory convection in the floating zone is mainly determined by the surface shear force, and the critical Marangoni number in the floating zone may be larger if the encapsulated liquid has a relatively larger viscosity in comparison with the floating zone liquid. The encapsulated column may be beneficial to the crystal growth by using the floating zone technique if the medium of encapsulated column is selected with larger viscosity.

6.3 Particle Accumulation Structure. The particle accumulation structure (PAS) is a special phenomenon of liquid/solid two phase flow in a floating zone, and appears as the particle pattern of solid state under certain ranges of parameters. The PAS may be observed in a narrow region of Marangoni number, nearly double the critical Marangoni number, and in a region of particle configuration and density, which is usually larger than the density of 


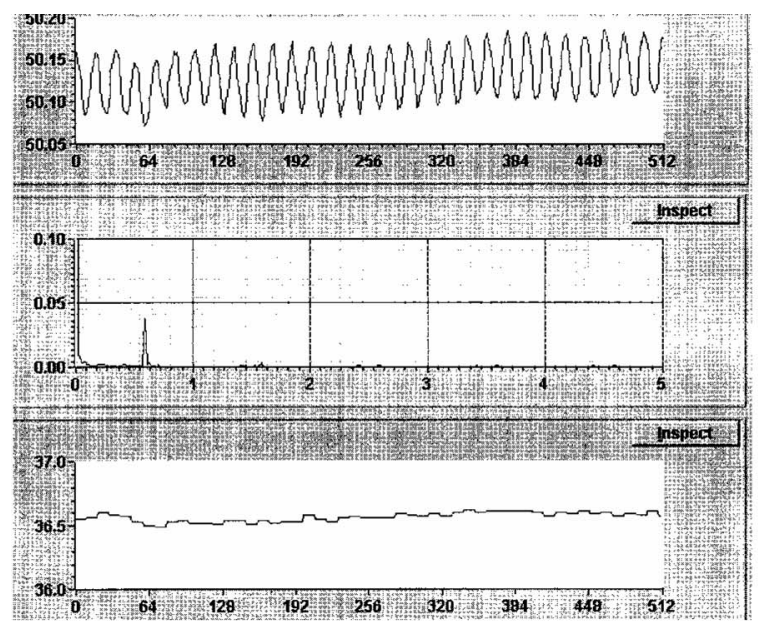

(a)

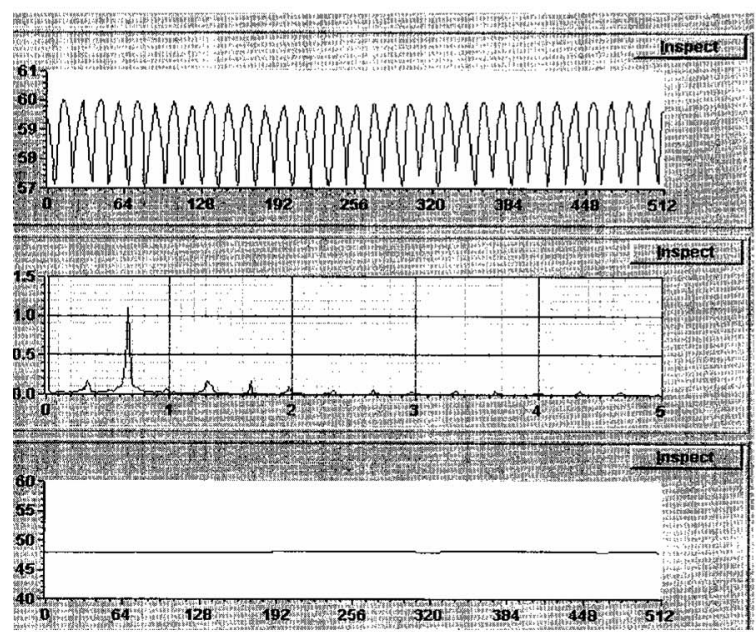

(b)

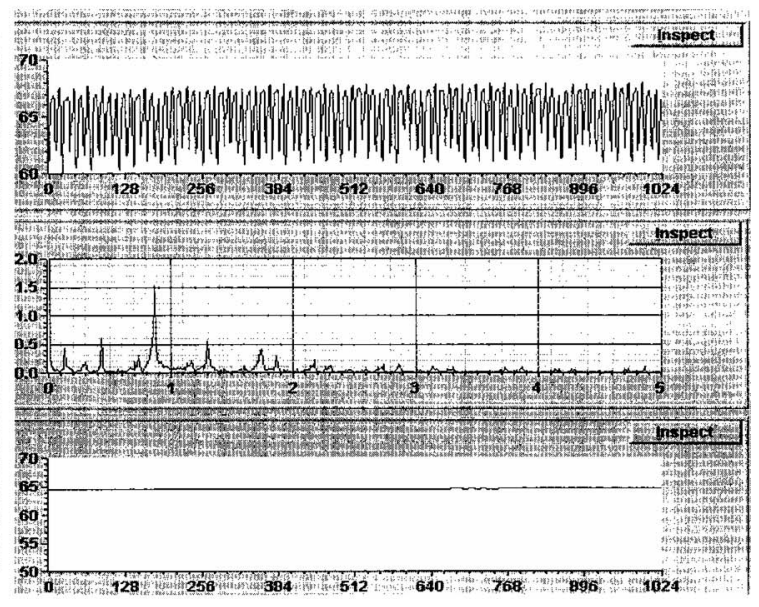

(d)

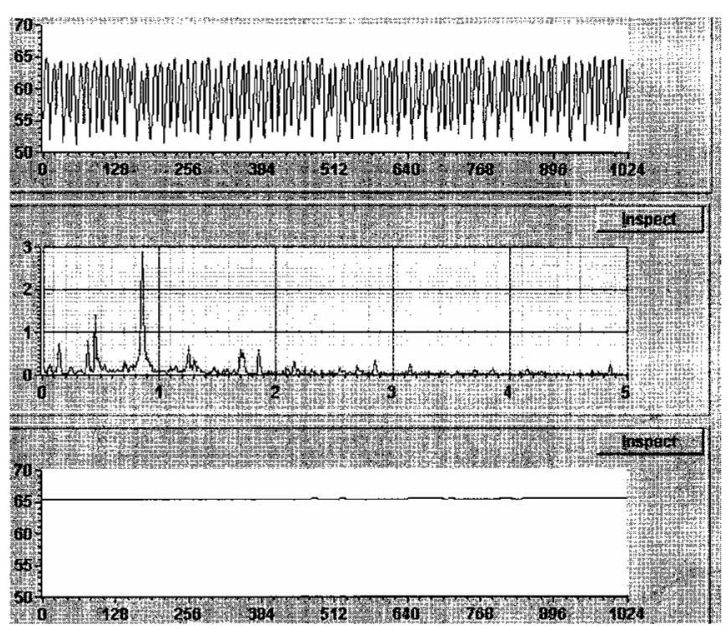

(e)

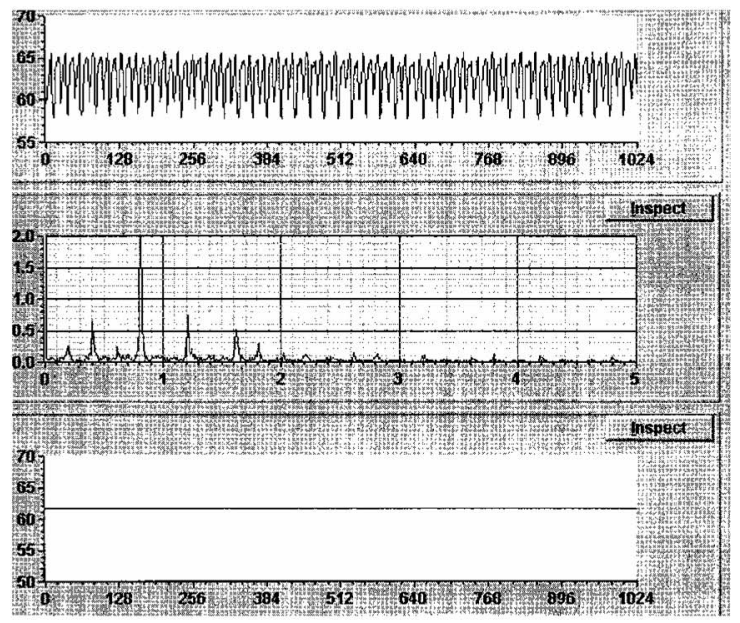

(c)

Fig. 8 The measured temperature distributions (above), spectra (middle), and applied temperature differences (lower) given by the experimental results. The subharmonic bifurcations appear at $(a), f_{0}=(36.55 \pm 0.05)^{\circ} \mathrm{C},(b)$ $f_{2}=(48.01 \pm 0.05)^{\circ} \mathrm{C}$, (c) $f_{4}=(61.65 \pm 0.05){ }^{\circ} \mathrm{C}$, (d) $f_{8}=(64.56 \pm 0.05)^{\circ} \mathrm{C}$, and $(e) f_{16}=(65.15 \pm 0.05){ }^{\circ} \mathrm{C}$, respectively. 
floating zone liquid. The experiments both on the ground and in space were performed [92,93], and the mechanism of the PAS needs to be studied in the future.

\section{Conclusion}

The studies of floating zone convection have been undertaken in the past few decades, and we continue to gain understanding of basic features in the transition process. Many phenomena on the onset of oscillatory thermocapillary convection are discovered by the experimental studies, and theoretical models have been proposed to describe the system. However, there are still many questions left for further investigations.

Several critical parameters, such as the Marangoni number, Prandtl number, and aspect ratio, have been the emphasis of many studies. In addition to the aspect ratio, the ratio of liquid bridge volume, another geometrical parameter, is analyzed recently, and experimental results may differ quite a bit for systems with identical aspect ratios but different ratios of liquid bridge volume. Moreover, the critical Marangoni number for a liquid bridge with a larger diameter of centimeters in the microgravity environment is much larger than the one with a small diameter of millimeters on the ground for the same aspect ratio and the ratio of liquid bridge volume. This implies that the dimensional scale of the liquid bridge is another geometrical critical parameter that needs to be considered together with the aspect ratio and the ratio of liquid bridge volume. Heat transfer dominates the floating zone process, and the applied temperature difference, or the Marangoni number, is a most important critical parameter. Every critical parameter represents an effect on the transition process, and the margin relationship for the onset of oscillatory thermocapillary convection is a complex function depending on the critical parameters.

The experimental results are essential for the understanding of the transition process. Due to the technical difficulties, there are limited test cases covering quantitative experiments of floating zone convection for the cases of small Prandtl number fluids, and the result data are dispersive. A few space experiments on thermocapillary convection in the floating half zone with large Prandtl number fluids were performed. Results from these space experiments indicate that the critical Marangoni number is larger and has a different dependency on the aspect ratio when compared with results from experiments conducted on the ground. Therefore, additional space experiments are required to further diagnose the mechanism of the transition process in thermocapillary convection. Extensive experiments have been devoted to the smallscale liquid bridge on the ground, where the Bond number is close to 1 , and the influence from buoyancy effect cannot be overlooked. It takes due diligence to use these ground experimental results in any attempt to come up with a theoretical model trying to explain quantitatively the features under microgravity conditions. One of the reasons here is that the earth's gravity has an obvious influence on the ground experiments even for a liquid bridge with a diameter of only a few millimeters.

Many theoretical models have been suggested to explain the onset of oscillatory thermocapillary convection, and many of them are based on the idea of fluid instability. Ostrach and co-workers disagreed with the idea of fluid instability, and they introduced a critical $S$ parameter related to the free surface deformation [70-72]. The onset of oscillatory thermocapillary convection is a complex process. Several criteria for the onset of oscillation have been suggested based on the simplified models of different mechanisms; however, a general criterion, such as that described by relationship (7), has not been obtained yet.

The route from oscillatory thermocapillary convection to the turbulence is a new frontier in the microgravity fluid physics, and a limited amount of research works have been spent on this subject. The scientific importance of this new area of research is tightly coupled with some topics in the nonlinear science, and we shall see more efforts devoted to these fields in the near future.

\section{Acknowledgment}

This work was supported by the National Natural Science Foundation of China (Contract No. 10432060) and the Knowledge Innovation Program of CAS.

\section{References}

[1] Ostrach, S., 1982, "Low-Gravity Fluid Flow," Annu. Rev. Fluid Mech., 14, pp. 313-345.

[2] 1987, Space Fluid Science and Materials Science, H. U. Walter, ed., ESA, Paris.

[3] Materials and Fluids Under Low Gravity: Proceedings of the IX European Symposium on Gravity-Dependent Phenomena in Physical Sciences, L. Ratke, H. Walter, and B. Feuerbacher, eds., Springer, Berlin.

[4] Jones, L. E., and Narayanan, R., 2002, Interface Instability, Springer, New York.

[5] Pearson, J. R. A., 1958, "On Convection Cells Induced by Surface Tension,” J. Fluid Mech., 4, pp. 489-500.

[6] Xiong, B., and Hu, W. R., 1992, "Crystal Growth in Floating Zone With Phase Change and Thermo-Solutal Convection," J. Cryst. Growth, 125, pp. 149-156.

[7] Chang, C. E., and Wilcox, W. R., 1975, "Inhomogeneities Due to Thermocapillary Flow in Floating Zone Melting," J. Cryst. Growth, 28, pp. 8-12.

[8] Chun, Ch. H., and Wuest, W., 1978, "A Micro-Gravity Simulation of the Marangoni Convection," Acta Astronaut., 5, pp. 681-686.

[9] Chun, Ch. H., and Wuest, W., 1979, "Experiments on the Transition From the Steady to the Oscillatory Marangoni-Convection of a Floating Zone Under Reduced Gravity Effect," Acta Astronaut., 6, pp. 1073-1082.

[10] Schwabe, D., Scharmann, A., Preisser, F., and Oeder, R., 1978, "Experiments on Surface Tension Driven Flow in Floating Zone Melting," J. Cryst. Growth, 43, pp. 305-312.

[11] Schwabe, D., and Scharmann, A., 1979, "Some Evidence for the Existence and Magnitude of a Critical Marangoni Number for the Onset of Oscillatory Flow in Crystal Growth Melts," J. Cryst. Growth, 46, pp. 125-131.

[12] Kuhlmann, H. C., 1999, Thermocapillary Convection in Model of Crystal Growth, Springer, New York.

[13] Hu, W. R., and Tang, Z. M., 2003, Floating Zone Convection, Science, Beijing.

[14] Levenstam, M., Amberg, G., and Wimkler, C., 2001, "Instabilities of Thermocapillary Convection in a Half-Zone at Intermediate Prandtl Numbers," Phys. Fluids, 13, pp. 807-816.

[15] Preisser, F., Schwabe, D., and Scharmann, A., 1983, "Steady and Oscillatory Thermocapillary Convection in Liquid Columns with Free Cylindrical Surface," J. Fluid Mech., 126, pp. 545-567.

[16] Hu, W. R., Shu, J. Z., Zhou, R., and Tang, Z. M., 1994, "Influence of Liquid Bridge Volume on the Onset of Oscillation in Floating Zone Convection I. Experiment," J. Cryst. Growth, 142, pp. 379-386.

[17] Tang, Z. M., and Hu, W. R., 1994, "Influence of Liquid Bridge Volume on the Onset of Oscillation in Floating Zone Convection II. Numerical Simulation, , J. Cryst. Growth, 142, pp. 385-391.

[18] Levenstam, M., and Amberg, G., 1995, "Hydrodynamical Instabilities of Thermocapillary Flow in Half-Zone," J. Fluid Mech., 297, pp. 357-372.

[19] Nakamura, S., Hibiya, T., Imaishi, N., Yoda, S., Nakamura, T., Koyama, M. Dold, P., and Benz, K. W., 1999, "Observation of Periodic Marangoni Convection in a Molten Silicon Bridge on Board the TR-IA-6 Rocket," J. Jpn. Soc. Microgravity Appl., 16, pp. 99-103.

[20] Chang, Y. K., 1983, "The Float-Zone Growth of $\mathrm{Ti}_{3} \mathrm{Au}$ and $\mathrm{Ti}_{3} \mathrm{Au}$," J. Cryst. Growth, 62, pp. 627-632.

[21] Croll, A., Muller-Sebert, W., and Nitche, R., 1989, “The Critical Marangoni Number for the Onset of Time-Dependent Convection in Silicon," Mater. Res. Bull., 24, pp. 995-1004.

[22] Jurisch, M., and Loser, W., 1990, "Analyses of Periodic Non-Rotational W Striation in Mo Single Crystals Due to Nonsteady Thermocapillary Convection," J. Cryst. Growth, 102, pp. 214-222.

[23] Jurish, M., 1990, "Surface Temperature Oscillatory of a Floating Zone Resulting From Oscillatory Thermocapillary Convection," J. Cryst. Growth, 102, pp. 223-232.

[24] Rupp, R., Muller, G., and Neumann, G., 1989, "Three-Dimensional and TimeDependent Modeling of the Marangoni Convection in a Zone Melting Configuration for GaAs," J. Cryst. Growth, 97, pp. 34-41.

[25] Croll, A., Kaiser, Th., Schweizer, A., Danilewsky, A. N., Lauer, S., Tegetmeier, A., and Benz, K. W., 1998, "Floating-Zone and Floating-Solutal Zone of GaSb Under Microgravity,” J. Cryst. Growth, 191, pp. 365-376.

[26] Han, J. H., Sun, Z. W., Dai, L. R., et al., 1996, "Experiment on the Thermocapillary Convection of a Mercury Liquid Bridge in a Floating Half Zone," J. Clim., 169, pp. 129-135.

[27] Sun, Z. W., Han, J. H., Dai, L. R., Xie, J. C., and Hu, W. R., 1997, "Experimental Study of Thermocapillary Convection on a Liquid Bridge Consisting of Fluid With Low Prandtl Number in a Floating Half-Zone," Science in China E., 29(1), pp. 97-104.

[28] Cao, Z. H., Xie, J. C., Tang, Z. M., and Hu, W. R., 1991, "The Influence of Buoyancy on the Onset of Oscillatory Convection in a Half Floating Zone," Adv. Space Res., 11(7), pp. 166-170.

[29] Cao, Z. H., You, H. T., Tang, Z. M., and Hu, W. R., 1991, "Experimental Investigation of Thermocapillary Convection in a Half Floating Zone," Adv Space Res., 11(7), pp. 232-236. 
[30] Yang, Y. K., and Kou, S., 2001, “Temperature Oscillation in a Tin Liquid Bridge and Critical Marangoni Number Dependency on Prandtl Number," J. Cryst. Growth, 222, pp. 135-143.

[31] Takagi, K., Otaka, M., Natsui, H., Arai, T., Yoda, S., Yuan, Z. F., Mukai, K., Yasuhiro, S., and Imaishi, N., 2001, "Experimental Study on Transition to Oscillatory Thermocapillary Flow in a Low Prandtl Number Liquid Bridge,” J. Cryst. Growth, 233, pp. 399-407.

[32] Schatz, M. F., and Neitzel, G. P., 2001, "Experiments on Thermocapillary Instability," Annu. Rev. Fluid Mech., 33, pp. 93-129.

[33] Velten, R., Schwabe, D., and Scharmann, A., 1991, "A Periodic Instability of Thermocapillary Convection in Cylindrical Liquid Bridge," Phys. Fluids A, 3, pp. 267-177.

[34] Chun, Ch. H., 1980, "Experiments on Steady and Oscillatory Temperature Distribution in a Floating Zone Due to the Marangoni Convection," Acta Astronaut., 7(4-5), pp. 479-488.

[35] Komotani, Y., Ostrach, S., and Vargas, M., 1984, "Oscillatory Thermocapillary Convection in a Simulated Floating-Zone Configuration," J. Cryst. Growth, 66, pp. 83-90.

[36] Xie, J. C., Shu, J. Z., Yao, Y. L., Tang, Z. M., and Hu, W. R., 1996, "The Oscillation Feature of Thermocapillary Convection,” Adv. Astronaut. Sci., 91, pp. 441-453.

[37] Hirata, A., Sakurai, M., Ohishi, N., and Koyama, M., 1997, "Transition Process From Laminar to Oscillatory Marangoni Convection in a Liquid Bridge," J. Jpn. Soc. Microgravity Appl., 14, pp. 137-143.

[38] Schwabe, D., Preisser, F., and Scharmann, A., 1982, "Verification of the Oscillatory State of Thermocapillary Convection in a Floating Zone Under Low Gravity," Acta Astronaut., 9, pp. 265-273.

[39] Schwabe, D., and Scharmann, A., 1984, "Microgravity Experiments on the Transition From Laminar to Oscillatory Thermocapillary Convection in Floating Zones," Adv. Space Res., 4(5), pp. 43-47.

[40] Monti, R., Fortezza, R., Desiderio, G., et al., 1992, “Onset of Oscillatory Marangoni Flow: Scientific Results of the Experiment Performed in Telescience on Texus, 23," Final Report of Sounding Rocket Experiments in Fluid Science and Material Science, ESA Report No. SP-1132, Vol. 2, pp. 40-64.

[41] Albanese, C., Carotenuto, L., Castagnolo, D., Ceglia, E., and Monti, R., 1995, "First Results From "Onset" Experiment During D2 Space Mission," in Scientific Results of German Spacelab Mission D-2, P. R. Sahm, M. H. Keller, and B. Schiewe, eds., DARA, Bonn, pp. 247-258.

[42] Carotenuto, L., Castagnolo, D., Albabese, C., and Monti, R., 1998, "Instability of Thermocapillary Convection in Liquid Bridge," Phys. Fluids, 10, pp. 555565.

[43] Monti, R., Castagnolo, D., Dell'Aversana, P., Desiderio, G., Moreno, S., and Evangelista, G., 1992, "An Experimental and Numerical Analysis of Thermocapillary Flow in Silicon Oils in a Micro-Floating Zone," 43rd Congress $I A F$, Washington, D.C.

[44] Sumner, L. B. S., and Neitzel, G. P., 2001, "Oscillatory Thermocapillary Convection in Liquid Bridges With Highly Deformed Free Surface," Phys. Fluids, 13, pp. 107-120.

[45] Frank, S., and Schwabe, D., 1992, "Temporal and Spatial Elements of Thermocapillary Convection in Floating Zones," Exp. Fluids, 23, pp. 234-251.

[46] Han, J. H., Zhou, Y. R., and Hu, W. R., 1997, "Investigation on a Simulation Model of Floating Half Zone Convection. II. Experiment," Int. J. Heat Mass Transfer, 40, pp. 2671-2677.

[47] Hu, W. R., You, H. T., and Cao, Z. H., 1992, "Free Surface Oscillation of Thermocapillary Convection in Liquid Bridge of Half Floating Zone," Scientia Sinica (Science in China), A35, pp. 1101-1107.

[48] Yao, Y. L., Liu, F., and Hu, W. R., 1996, "How to Determine Critical Marangoni Number in Half Floating Zone Convection,” Int. J. Heat Mass Transfer, 39, pp. 2539-2544.

[49] Shu, J. Z., Yao, Y. L., and Hu, W. R., 1993, "Free Surface Vibration in Oscillatory Convection in Half Floating Zone," Scientia Sinica (Science in China), A36, pp. 326-332.

[50] Yao, Y. L., Shu, J. Z., Xie, J. C., Hu, W. R., Hirata, A., Nishizawa, S. I., and Sakurai, M., 1997, "Transition of Oscillatory Floating Half Zone Convection From Earth's Gravity to Microgravity," Int. J. Heat Mass Transfer, 40, pp. 2517-2523.

[51] Hu, W. R., and Tang, Z. M., 2000, "Onset Process of Thermocapillary Oscillatory Convection," Microgravity and Space Station Utilization (Microgravity Quarterly), 1, pp. 23-30.

[52] Tang, Z. M., Aa, Y., Cao, Z. H., and Hu, W. R., 2002, "Two Bifurcation Transition Processes in Floating Half Zone Convection of Larger Prandtl Number Fluid," Acta Mech. Sin., 18, pp. 328-336.

[53] Hu, W. R., and Tang, Z. M., 2003, "Influence of Liquid Bridge Volume on the Floating Zone Convection," Cryst. Res. Technol., 38, pp. 627-634.

[54] Aa, Y., Cao, Z. H., Tang, Z. M., and Hu, W. R., 2005, "Experimental Study on the Transition Process to the Oscillatory Thermocapillary Convection in a Floating Half Zone, Microgravity Science and Technology," Microgravity Sci. Technol., 17(4), pp. 5-12.

[55] Smith, M. K., and Davis, S. H., 1983, "Instabilities of Dynamic Thermocapillary Liquid Layer. Part I. Convective Instabilities," J. Fluid Mech., 132, pp. 119-131.

[56] Smith, M. K., and Davis, S. H., 1983, "Instabilities of Dynamic Thermocapillary Liquid Layer. Part 2. Surface Wave Instability," J. Fluid Mech., 132, pp. 132-144.

[57] Xu, J. J., and Davis, S. H., 1984, "Convective Thermocapillary Instabilities in Liquid Bridge,” Phys. Fluids, 27, pp. 1102-1107.

[58] Chen, G., Lizee, A., and Roux, B., 1997, "Bifurcation Analysis of Thermocap- illary Convection in Cylindrical Liquid Bridges,” J. Cryst. Growth, 180, pp. 638-647.

[59] Neitzel, G. P., Chang, K. T., Jankowski, D. F., and Mittelmann, H. D., 1993, "Linear Stability Theory of Thermocapillary Convection in a Model of the Float-Zone Crystal-Growth Process," Phys. Fluids A, 5, pp. 108-114.

[60] Kuhlmann, H. C., and Rath, H. J., 1993, "Hydrodynamic Instability in Cylindrical Thermocapillary Liquid Bridges," J. Fluid Mech., 247, pp. 247-274.

[61] Wanschura, M., Shevtsova, V. M., Kuhlmann, H. C., and Rath, H. J., 1995, "Convective Instability Mechanisms in Thermocapillary Liquid Bridge," Phys. Fluids, 7, pp. 912-925.

[62] Sim, B. C., and Zebib, A., 2004, "Thermocapillary Convection in Cylindrical Liquid Bridge and Annuli," C. R. Mec., 332, pp. 473-486.

[63] Chen, Q. S., and Hu, W. R., 1998, "Influence of Liquid Bridge Volume on Instability of Floating Half Zone Convection,” Int. J. Heat Mass Transfer, 41, pp. 825-837.

[64] Chen, Q. S., Hu, W. R., and Prasad, V., 1999, "Effect of Liquid Bridge Volume on the Instability in Small-Prandtl-Number Half Zone," J. Cryst. Growth, 203, pp. 261-268.

[65] Nienhuser, C. H., and Kuhlmann, H. C., 2002, "Stability of Thermocapillary Flows in Non-Cylindrical Liquid Bridge," J. Fluid Mech., 458, pp. 35-74.

[66] Shen, Y., Neitzed, G. P., Jankowski, D. F., and Mittelmann, H. D., 1990, "Energy Instability of Thermocapillary Convection in a Model of the FloatingZone Crystal Growth Process," J. Fluid Mech., 217, pp. 639-660.

[67] Neitzed, G. P., Law, C. C., Jankowski, D. F., and Mittelmann, H. D., 1991, "Energy Stability of Thermocapillary Convection in a Model of Floating-Zone Crystal-growth Process. II: Nonaxisymmetric Disturbances," Phys. Fluids A, 3, pp. 2841-2846.

[68] Smith, M. K., 1986, "Instability Mechanisms in Dynamic Thermocapillary Liquid Layer," Phys. Fluids, 29, pp. 3182-3186.

[69] Davis, S. H., 1987, "Thermocapillary Instability," Annu. Rev. Fluid Mech., 19, pp. 403-435.

[70] Kamotani, Y., and Ostrach, S., 1998, "Theoretical Analysis of Thermocapillary Flow in Cylindrical Columns of High Prandtl Number Fluids," ASME J. Heat Transfer, 120, pp. 758-767.

[71] Kamotani, Y., and Ostrach, S., 1999, "Oscillatory Thermocapillary Flows in Open Cylindrical Containers Induced by $\mathrm{CO}_{2}$ Laser Heating," Int. J. Heat Mass Transfer, 42, pp. 555-564.

[72] Kamotani, Y., Ostrach, S., and Pline, A., 1994, "Analysis of Velocity Data Taken in Surface Tension Driven Convection Experiment in Microgravity," Phys. Fluids, 6, pp. 3601-3609.

[73] Shevtosova, V., 2005, "Thermal Convection in Liquid Bridge With Curved Free Surface: Benchmark of Numerical Solution," J. Cryst. Growth, 280, pp. 632-651.

[74] Savino, R., and Monti, R., 1996, "Oscillatory Marangoni Convection in Cylindrical Liquid Bridge," Phys. Fluids, 8, pp. 2906-2922.

[75] Leypoldt, J., Kuhlmann, H. C., and Rath, H. J., 2000, "Three Dimensional Numerical Simulation of Thermocapillary Flows in Cylindrical Liquid Bridges," J. Fluid Mech., 414, pp. 285-314.

[76] Tang, Z. M., and Hu, W. R., 1999, "Influence of Liquid Bridge Volume on the Onset of Oscillation in Floating Zone Convection. III. Three Dimensional Model," J. Cryst. Growth, 207, pp. 239-246.

[77] Tang, Z. M., Hu, W. R., and Imaishi, N., 2001, "Two Bifurcation Transitions of the Floating Half Zone in a Fat Liquid Bridge of Large Pr,” Int. J. Heat Mass Transfer, 44, pp. 1299-1307.

[78] Yasushiro, S., Imaishi, N., Akiyama, Y., Fujino, S., and Yoda, S., 2004, "Oscillatory Marangoni Flow in Half-Zone Liquid Bridge of Molten Tin," J. Cryst. Growth, 262, pp. 631-644.

[79] Yasushiro, S., Li, K., Imaishi, N., Akiyama, Y., Natsui, H., Matsumoto, S., an Yoda, S., 2004, "Oscillatory Thermocapillary Flow in a Half-Zone Liquid Bridge of Molten Tin," J. Cryst. Growth, 266, pp. 152-159.

[80] Li, K., Yasushiro, S., Imaishi, N., and Yoda, S., 2005, "Marangoni Flow in Half-Zone Liquid Bridge of Molten Tin Under Ramped Temperature Difference," J. Cryst. Growth, 280, pp. 620-631.

[81] Feigenbaum, M. J., 1979, "The Onset Spectrum of Turbulence," Phys. Lett. 74A, pp. 375-378.

[82] Chun, Ch. H., 1984, "Verification of Turbulence Developing From the Oscillatory Marangoni Convection in a Liquid Column," Eur. Space Agency, [Spec. Publ.] ESA SP, ESA SP-222, pp. 271-280.

[83] Tang, Z. M., and Hu, W. R., 1995, "Fractal Feature of Oscillatory Convection in Half-Floating Zone," Int. J. Heat Mass Transfer, 38, pp. 3295-3300.

[84] Aa, Y., Cao, Z. H., and Hu, W. R., 2004, "Transition to Turbulence in the Floating Half Zone Convection," 54th International Astronautic Congress, Bremen, Germany, Oct. 2003, Paper No. IAC-03-J.4.06.

[85] Schwabe, D., and Frank, S., 1999, "Experiments on the Transition to Chaotic Thermocapillary Flow in Floating Zones Under Microgravity," Adv. Space Res., 24(10), pp. 1391-1396.

[86] Kamotani, Y., Wang, L., Hatta, S., Wang, A., and Yoda, S., 2003, "Free Surface Heat Loss Effect on Oscillatory Thermocapillary Flow in Liquid Bridge of High Prandtl Number Fluids," Int. J. Heat Mass Transfer, 46, pp. 32113220.

[87] Selver, R., 2005, "Experiments on the Transition From the Steady to the Oscillatory Marangoni Convection of a Floating-Zone Under Various Cold Wall Temperature and Various Ambient Air Temperature Effects," Microgravity Sci. Technol., 17, pp. 25-35.

[88] Kousaka, Y., and Kawamura, H., 2006, "Numerical Study on the Effect of Heat Loss Upon the Critical Marangoni Number in a Half-Zone Liquid Bridge," Microgravity Sci. Technol., 18(3-4) pp. 141-145. 
[89] Melinikov, D. E., and Shevtsova, V. M., 2006, "Thermocapillary Convection in a Liquid Bridge Subjected to Interfacial Cooling," Microgravity Sci. Technol., 18(3-4) pp. 128-131.

[90] Doi, T., and Koster, N., 1993, "Thermocapillary Convection in a Two Immiscible Two Layers With Free Surface," Phys. Fluids A, 5, pp. 1914-1921.

[91] Majima, S., Kawamura, H., Otsubo, F., Kuwahara, K., and Doi, T., 2001, "Oscillatory Thermocapillary Flow in Encapsulated Liquid Column," Phys. Fluids, 13, pp. 1517-1520.
[92] Tanaka, S., Kawamura, H., Ueno, I., and Schwabe, D., 2006, "Flow Structure and Dynamic Particle Accumulation in Thermocapillary Convection in a Liquid Bridge," Phys. Fluids, 18, pp. 067103-067103.

[93] Schwabe, S., Tanaka, S., Mizev, A., and Kawamura, H., 2006, "Particle Accumulation Structure in Time-Dependent Thermocapillary Flow in a Liquid Bridge Under Microgravity," Microgravity Sci. Technol., 18(3-4) pp. 117127.
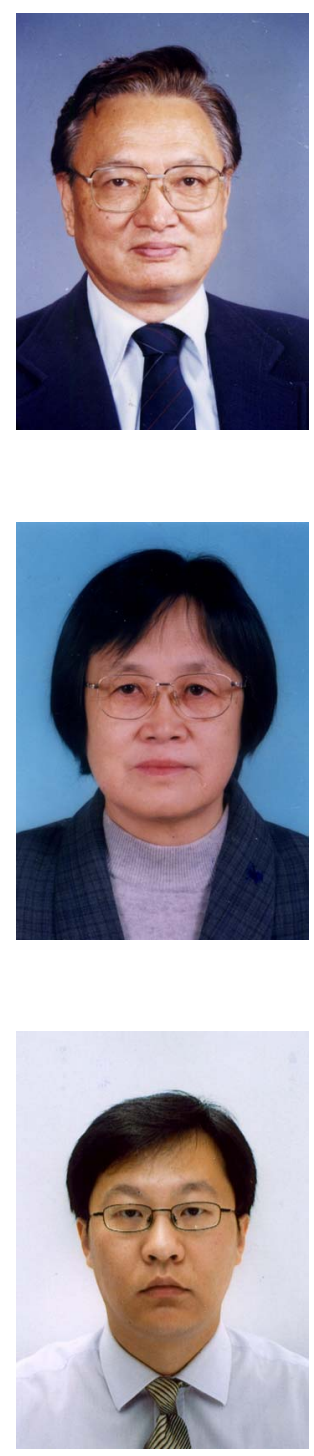

Wen-rui Hu is an academician of the Chinese Academy of Sciences. Professor Hu has been a full member of the International Astronautic Academy since 1996. He graduated from the Department of Mathematics and Mechanics, Peking University in 1958 and then worked in the Institute of Mechanics of the Chinese Academy of Sciences as an assistant (1958-1978), associate professor (1978-1984), and full professor (1984-present). His research work has been on the microgravity fluid physics, especially in the field of thermocapillary convection and drop migration since late 1980s. He has authored ten books and more than 200 article length publications.
Zei-mei Tang is a professor in the Institute of Mechanics, Chinese Academy of Sciences. She graduated from Tsing Hua University in 1965. She has done research numerically by using finite element method in the field of thermocapillary convection since late of 1980s. Professor Tang has authored more than 50 article publications on microgravity fluid physics.
Kai Li is a professor in Institute of Mechanics, Chinese Academy of Sciences (CAS). After getting his Ph.D. degree from the Institute of Mechanics, CAS, he worked as a research associate in Washington State University (USA) and as an assistant professor in Kyushu University (Japan). His major research interests are the numerical studies of thermocapillary flow instability in half zone configuration and heat and mass transfer process in crystal growth systems on ground and in microgravity, such as floating zone method and Bridgman method. He has authored more than 20 article publications on microgravity fluid physics. 